\title{
UNE ESTIMATION \\ DES COEFFICIENTS TANGENTS \\ D'UN COURANT POSITIF FERME \\ DANS UN DOMAINE DE $\mathbb{C}^{3}$
}

Ph. Charpentier and Y. Dupain

Abstract

In this paper, we study the behaviour ncar the boundary of the complex tangent coefficients of a closed positive current in a bounded domain of $\mathbb{C}^{3}$ with $\mathcal{C}^{\infty}$ boundary. Assuming that the current satisfies the Blaschke condition, we give a condition on the complex tangent coefficients which is better than the one which can be proved using the pseudo-distance introduced by A. Nagel, $\mathrm{E}$. Stein and $\mathrm{S}$. Wainger (in analogy with the case of domains in $C^{2}$ ). Moreover, when the domain is supposed to be pseudoconvex, we show how our condition is related to $\mathrm{D}$. Catlin's multitype.

\section{Introduction}

Soit $\Omega$ un domaine borné de $\mathbb{C}^{n}$ à frontière de classe $\mathcal{C}^{\infty}$, et soit $\rho \in$ $\mathcal{C}^{\infty}\left(\mathbb{C}^{n}\right)$ une fonction définissante de $\Omega$. Soit $\theta=\sum_{i, j} \theta_{i, j} d z_{i} \wedge d \bar{z}_{j}$ un $(1,1)$-courant positif fermé (i.e. $d \theta=0$ ) dans $\Omega$ satisfaisant à la condition de Blaschke, c'est-à-dire tel que

$$
\int_{\Omega}(-\rho) \sum_{i} \theta_{i i}<+\infty
$$

Il est classique que sous cette hypothèse, les coefficients tangents de $\theta$, c'est-à-dire les coefficients de la forme $\theta \wedge \partial \rho \wedge \bar{\partial} \rho$, satisfont à une condition meilleure connue sous le nom de condition de Malliavin: précisément, on a

$$
\int_{\Omega}|\theta \wedge \partial \rho \wedge \vec{\partial} \rho| \leq C \int_{\Omega}(-\rho)|\theta|
$$


où $C$ ne dépend que $\Omega$, ct $|\theta \wedge \partial \rho \wedge \bar{\partial} \rho|$ désigne la somme des modules des coefficients de la forme.

Cette condition n'est optimale que dans le cas des domaines strictement pseudoconvexes (c.f. [2]), et des conditions meilleures ont été données dans deux situations distinctes. Tout d'abord dans le cas des $e l$ lipsoïdes complexes, il est montré dans [1] que les coefficients de $\theta \wedge \partial \rho \wedge \bar{\partial} \rho$ satisfont une condition strictement meilleure lorsque l'ellipsoìde n'est pas la boule. D'un autre côté, dans [2] on étudie le cas des domaines de $\mathbb{C}^{2}$. On y démontre que l'on a une condition de la forme

$$
\int_{\Omega} \frac{|\theta \wedge \partial \rho \wedge \bar{\partial} \rho|}{S} \leq \int_{\Omega}(-\rho)|\theta|
$$

où la fonction $S$ cst lićc à la pscudo-distance introduite par A. Nagel, E. Stein et $S$. Wainger dans [5] et [6], c'est-à-dire essentiellement au type de Kohn des points du domaine. En particulier, si $\Omega$ est de type fini non strictement pseudoconvexe, la condition obtenue est meilleure que la condition de Malliavin. De plus, le travail postérieur de D.C. Chang, A. Nagel, et $\mathrm{E}$. Stein [4] a montré que cette condition était la bonne.

Il est facile de voir que cette condition reste valable dans un domaine quelconque de $\mathbb{C}^{n}$. Mais l'exemple des ellipsoïdes complexes montre qu'alors elle n'est plus optimale lorsque $n \geq 3$.

Dans ce travail, nous nous plaçons dans $\mathbb{C}^{3}$, et nous obtenons une condition sur les coefficients tangents du courant qui est, meilleure que la condition précédente. Pour être un peu plus précis, soit $z_{0}$ un point de la frontière de $\Omega$, et soit $\mathcal{B}=\left(L_{1}, L_{2}\right)$ une base de l'espace des $(1,0)$ champs tangents complexes au voisinage de $z_{0}$ de sorte que, si $N$ est le champ normal à $\rho$. (i.e. $N \rho \equiv 1$ au voisinage de la frontière de $\Omega$ ), $\left(N, L_{1}, L_{2}\right)$ est une base des champs holomorphes au voisinage de $z_{0}$, et soit $\left(\partial \rho, \omega_{1}, \omega_{2}\right)$ la base duale de $(1,0)$-formes. Nous contruisons alors deux fonctions $S_{1}$ et $S_{2}$ dans un voisinage $V$ de $z_{0}$ telles que

$\int_{V \cap \Omega} \frac{\left|\theta \wedge \partial \rho \wedge \bar{\partial} \rho \wedge \omega_{2} \wedge \bar{\omega}_{2}\right|}{S_{1}}+\int_{V \cap \Omega} \frac{\left|\theta \wedge \partial \rho \wedge \bar{\partial} \rho \wedge \omega_{1} \wedge \bar{\omega}_{1}\right|}{S_{2}} \leq \int_{\Omega}(-\rho)|\theta|$,

la constante $C$ ne dépendant que de $\Omega, V$ et $B$.

Les fonctions $S_{1}$ et $S_{2}$, dont les défnitions précises sont données au paragraphe suivant, sont inférieures à la fonction $S$ de l'inégalité (3), et ne sont plus en fait liées uniquement au type de Kohn des points du domaine $\Omega$.

Un peu plus précisément, au paragraphe $V$, nous comparons leurs ordres de croissance au bord de $\Omega$ au multitype introduit par D. Catlin dans [3]. Disons simplement ici que lorsque l'on choisit pour $\mathcal{B}$ la base de 
champs associée au multitype de $z_{0}$, le comportement radial de $S_{1}$ et $S_{2}$ peut se comparer au multitype $\left(1, \lambda_{2}, \lambda_{3}\right)$ de $z_{0}$ (celui-ci étant supposé fini): $S_{1}$ est équivalent à $(-\rho)^{1-2 / \lambda_{2}}$ ct $S_{2}$ est inférieur à $(-\rho)^{1-2 / \lambda_{3}}$.

\section{Notations et énoncé du Théorème}

Soient $\Omega$ un domaine borné de $\mathbb{C}^{3}$ à bord $\mathcal{C}^{\infty}$ et $\rho$ une fonction définissante de $\Omega$ (i.e. $\nabla \rho \neq 0$ au voisinage de $\partial \Omega$ ). Soit $N$ lc $(1,0)$ champ normal à $\rho$ dans un voisinage de $\partial \Omega$ c'est-à-dire tel que $N \rho \equiv 1$ au voisinage de $\partial \Omega$ (i.e.

$$
\left.N=\frac{4}{|\nabla \rho|^{2}} \sum \frac{\partial \rho}{\partial \bar{z}_{i}} \frac{\partial}{\partial z_{i}}\right)
$$

Soit $z_{0}$ un point de $\partial \Omega$ fixć une fois pour toutes. Soit $\mathcal{B}=\left(L_{1}, L_{2}\right)$ une base de champs tangents à $\rho$ (i.e. $L_{i} \rho \equiv 0$ ) dans un voisinage de $z_{0}$. Soit $\left(\partial \rho, \omega_{1}, \omega_{2}\right)$ la base de $(1,0)$-formes, duale de la base de champs $\left(N, L_{1}, L_{2}\right)$

Soient $m$ et $M$ deux réels, $m$ entier, $2 \leq m \leq M$.

Reprenant les notations introduites par $\mathrm{D}$. Catlin dans $[3]$, la base $\mathcal{B}$ étant fixée, considérons la famille de listes suivante:

$$
W(\mathcal{B})=\left\{\mathcal{L}_{\mathcal{B}}=\mathcal{L}=\left(L^{1}, L^{2}, \ldots, L^{k}\right) ; k \geq 2, L^{i} \in\left\{L_{1}, \bar{L}_{1}, L_{2}, \bar{L}_{2}\right\}, \forall i\right\} .
$$

Pour chaque $\mathcal{L} \in W(\mathcal{B})$, nous considérons les paramètres suivants:

$$
\begin{aligned}
& l_{1}(\mathcal{L})=l_{1}=\operatorname{card}\left\{i \text { tel que } L^{i} \in\left\{L_{1}, \bar{L}_{1}\right\}\right\}, \\
& l_{2}(\mathcal{L})=l_{2}=\operatorname{card}\left\{i \text { tel que } L^{i} \in\left\{L_{2}, \bar{L}_{2}\right\}\right\},
\end{aligned}
$$

de sorte que $|\mathcal{L}|=k=l_{1}+l_{2}$

$m$ et $M$ étant fixés, définissons maintenant des sous-ensembles de $W(B):$

$$
\begin{aligned}
& W\left(L_{2}, \mathcal{B}, m, M\right)=W=\left\{\mathcal{L} \in W(\mathcal{B}) ; \frac{l_{1}}{m}+\frac{l_{2}}{M}<1\right\} ; \\
& W_{0}\left(L_{1}, m\right)=W_{0}=\left\{\mathcal{L} \in W ; l_{2}=0\right\} ; W_{1}(\mathcal{B}, m)=W_{1}=\left\{\mathcal{L} \in W ; l_{1}+l_{2}<m\right\} ; \\
& W_{2}\left(L_{2}, \mathcal{B}, m, M\right)=W_{2}=\left\{\mathcal{L} \in W ; l_{2} \geq 1\right\} \\
& W_{3}\left(L_{2}, \mathcal{B}, m, M\right)=W_{3}=\left\{\mathcal{L} \in W ; l_{1} \geq 1\right\} ; \\
& W_{4}\left(L_{2}, m, M\right)=W_{4}=\left\{\mathcal{L} \in W ; l_{1}=0, l_{2}<\frac{M}{m}\right\} .
\end{aligned}
$$


Pour $\mathcal{L} \in W$, nous définissons, suivant D. Catlin, $c_{L^{2}, \mathcal{B}, m}(\mathcal{L})=c$, par $\frac{l_{1}}{m}+\frac{l_{2}}{c}=1$.

Dans toute la suite, l'ensemble de listes $W_{4}$ jouera un rôle très particulier. Afin de rendre plus lisible cette particularité, tous les éléments de $W_{4}$, et seulement eux, seront notés $\widetilde{\mathcal{L}}$ au lieu de $\mathcal{L}$, et le paramètre correspondant sera noté $\tilde{l}_{2}$.

Pour définir les poids affectés aux coefficients du courant, il nous faut encore préciser une notation:

- Pour $\mathcal{L}=\left(L^{1}, \ldots, L^{k}\right) \in W(\mathcal{B})$, on note

$$
\mathcal{L}(\partial \rho)=L^{1} \ldots L^{k-2}\left(\partial \rho\left(\left[L^{k-1}, L^{k}\right]\right)\right)
$$

si $k>2$, et, $\mathcal{L}(\partial \rho)=\partial \rho\left(\left[L^{1}, L^{2}\right]\right)$ si $k=2$.

- Pour $\widetilde{\mathcal{L}}=\left(L^{1}, \ldots, L^{k}\right) \in W_{4}$ et pour $f$ une fonction $\mathcal{C}^{\infty}$ dans un voisinage de $z_{0}$, on note $\widetilde{\mathcal{L}}(f)=L^{1} \ldots L^{k-2}(f)$, si $k>2$, et, $\widetilde{\mathcal{L}}(f)=f$ si $k=2$.

- Enfin, nous notons $\gamma_{L_{2}, s}=\gamma$ le coefficient de $L_{1}$ dans le décomposition de $\left[L_{2}, \bar{L}_{2}\right]$ sur la base ( $\left(\Im m N, L_{1}, \bar{L}_{1}, L_{2}, \bar{L}_{2}\right)$.

Définissons maintenant des poids associés à la base $\mathcal{B}=\left(L_{1}, L_{2}\right)$ :

$$
S(\mathcal{B}, m)=\sum_{\mathcal{L} \in W_{1}}|\mathcal{L}(\partial \rho)|^{\frac{2}{k}}(-\rho)^{1-\frac{2}{k}}+(-\rho)^{1-\frac{2}{m}},
$$

et,

$$
S\left(L_{2}, \mathcal{B}, m, M\right)=S^{0}+S^{2}+S^{4}+S^{43}+(-\rho)^{1-\frac{2}{M}},
$$

où l'on a noté succesivement

$$
\begin{aligned}
& S^{0}=\sum_{\mathcal{L} \in W_{0}}|\mathcal{L}(\partial \rho)|^{\frac{2}{k}}(-\rho)^{1-\frac{2}{k}+\frac{2}{m}-\frac{2}{M}}, \\
& S^{2}=\sum_{\mathcal{L} \in W_{2}}|\mathcal{L}(\partial \rho)|^{\frac{2}{l_{2}}}(-\rho)^{1-\frac{2}{c}}, \\
& S^{4}=\sum_{\widetilde{\mathcal{L}} \in W_{4}}|\tilde{\mathcal{L}}(\gamma)|^{\frac{2}{i_{2}}}(-\rho)^{1-\frac{2}{m i_{2}}},
\end{aligned}
$$

et,

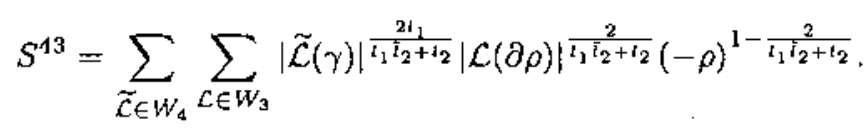

Remarquons que, si $M \leq 2 m, W_{4}$ est vide ct $S\left(L_{2}, \mathcal{B}, m, M\right)$ sc réduit à $S^{0}+S^{2}+(-\rho)^{1-2 / M}$. 
Nous définissons maintenant le poids $S\left(L_{2}, B, M\right)$ par:

$$
S\left(L_{2}, \mathcal{B}, M\right)=\inf \left\{S(\mathcal{B}, M), S\left(L_{2}, \mathcal{B}, m, M\right), 1 \leq m \leq M\right\} .
$$

En échangeant les rôles de $L_{1}$ et $L_{2}$, on définit de la même manière le poids

$$
S\left(L_{1}, \mathcal{B}, M\right)=\inf \left\{S(\mathcal{B}, M), S\left(L_{1}, \mathcal{B}, m, M\right), 1 \leq m \leq M\right\} .
$$

Rappelons enfin que, si $\theta$ est un $(1,1)$-courant positif dans $\Omega$, alors pour toutes $(1,0)$-formes à coefficients continus dans $\bar{\Omega}, \alpha_{1}$ et $\alpha_{2}, \theta \wedge i \alpha_{1} \wedge \bar{\alpha}_{1} \wedge i \alpha_{2} \wedge \bar{\alpha}_{2}$ est une mesure positive dans $\Omega$. De plus, le courant $i \theta \wedge \alpha_{1} \wedge \bar{\alpha}_{1}$ est positif.

Nous pouvons maintenant. énoncer le théorème:

\section{Théorème.}

Soit $\Omega$ un domaine bormé de $\mathbb{C}^{3} \grave{a}$ bord $\mathcal{L}^{\infty}$, et soit $\rho$ une fonction définissante de $\Omega$ (i.e. $\nabla \rho \neq 0$ au voisinage de $\partial \Omega$ ). Soient $z_{0}$ un point de $\partial \Omega$, et $\mathcal{B}=\left(L_{1}, L_{2}\right)$ une base de $(1,0)$ champs complexes tangents à $\rho$ au voisinage de $z_{0}$ (i.e. $L_{i}(\rho) \equiv 0$ ). Pour tout réel $M \geq 2$, il existe un réel $r>0$ et une constante $C>0$ dépendant de $\Omega$, de la base $\mathcal{B}$, de $M$ et de $r$ tels que:

Pour tout courant positif fermé $\theta$ dans $\Omega$ de bidegré $(1,1)$, l'inégalité suivante est vérifiée:

$$
\begin{aligned}
& \int_{\Omega \cap B\left(z_{0}, r\right)} \frac{\left|\theta \wedge \partial \rho \wedge \bar{\partial} \rho \wedge \omega_{2} \wedge \bar{\omega}_{2}\right|}{S\left(L_{1}, \mathcal{B}, M\right)}+ \\
+ & \int_{\Omega \cap B\left(z_{0}, r\right)} \frac{\left|\theta \wedge \partial \rho \wedge \bar{\partial} \rho \wedge \omega_{1} \wedge \bar{\omega}_{1}\right|}{S\left(L_{2}, \mathcal{B}, M\right)} \leq C \int_{\Omega}(-\rho)|\theta|,
\end{aligned}
$$

où, $\left(\partial \rho, \omega_{1}, \omega_{2}\right)$ est la base de $(1,0)$-formes duale de la base $\left(N, L_{1}, L_{2}\right)$, $N$ étant le champ normal à $\rho$ (i.e. $N \rho \equiv 1$ au voisinage de $\partial \Omega$ ).

\section{Remarque 1: une autre écriture des poids.}

Nous avons dit dans l'introduction que le théorème ci-dessus améliore le résultat que l'on peut obtenir, de façon analogue au cas de $\mathbb{C}^{2}$, en utilisant la pseudo-distance introduite dans [5] et [6]. Rappelons tout d'abord celui-ci. Avec les notations introduites précédemment, posons, pour $\delta>0$,

$$
R_{M}(\delta)=\inf _{2 \leq|\mathcal{L}| \leq \leq M}\left\{\left(\frac{\delta}{|\mathcal{L}(\partial \rho)|}\right)^{\frac{1}{|\mathcal{L}|}}, \delta^{1 / M}\right\}
$$


où, $|\mathcal{L}|=k$ désigne la longueur de la liste $\mathcal{L}$. Le résultat lié à la pseudodistance de [5] et [6] s'écrit:

$$
\int_{\Omega \cap B\left(\mathbf{z}_{0}, r\right)} \frac{\left[R_{M}(\delta(z))\right]^{2}}{\delta(z)}|\theta \wedge \partial \rho \wedge \bar{\partial} \rho| \leq C \int_{\Omega}(-\rho)|\theta|,
$$

où, $\delta(z)$ désigne la distance de $z$ à $\partial \Omega$.

Exprimons l'inégalité du théorème de manière analogue. Pour cela posons successivement

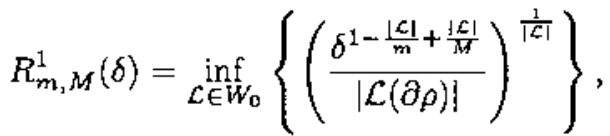

$$
\begin{aligned}
& R_{m, M}^{2}(\delta)=\inf _{\mathcal{L} \in W_{2}}\left\{\left(\frac{\delta^{1-\frac{t_{1}}{m}}}{|\mathcal{L}(\partial \rho)|}\right)^{\frac{1}{t_{2}}}\right\} \\
& R_{m, M}^{3}(\delta)=\inf _{\tilde{\mathcal{C}} \in W_{1}}\left\{\left(\frac{\delta^{\frac{3}{m}}}{|\widetilde{\mathcal{L}}(\gamma)|}\right)^{\frac{1}{i \mathcal{L} \mid}}\right\} \text {, } \\
& R_{m, M}^{4}(\delta)=\inf _{\tilde{\mathcal{L}} \in W_{4}, L \in W_{3}}\left\{\left(\frac{\delta}{|\overline{\mathcal{L}}(\gamma)|^{t_{1}}|\mathcal{L}(\partial \rho)|}\right)^{\frac{1}{\overline{1}_{1} i_{2}+i_{2}}}\right\}, \\
& R_{m, M}\left(L_{2}, \mathcal{B}\right)(\delta)=\inf _{1 \leq i \leq 4}\left\{R_{m, M}^{i}(\delta), \delta^{1 / M}\right\} \text {, }
\end{aligned}
$$

et,

$$
R_{M}\left(L_{2}, \mathcal{B}\right)(\delta)=\sup _{2 \leq m \leq M}\left\{R_{m, M}(\delta), R_{M}(\delta)\right\}
$$

De la même manière, définissons $R_{M}\left(L_{1}, \mathcal{B}\right)(\delta)$, en échrangeant les rôles de $L_{1}$ et $L_{2}$. Le théorème est alors équivalent à l'inégalité suivarite:

$$
\begin{aligned}
& \int_{\Omega \cap B\left(z_{0}, r\right)} \frac{\left[R_{M}\left(L_{1}, \mathcal{B}\right)(\delta(z))\right]^{2}}{\delta(z)}\left|\theta \wedge \partial \rho \wedge \bar{\partial} \rho \wedge \omega_{2} \wedge \bar{\omega}_{2}\right|+ \\
& +\int_{\Omega \cap B\left(z_{0}, r\right)} \frac{\left[R_{M}\left(L_{2}, \mathcal{B}\right)(\delta(z))\right]^{2}}{\delta(z)}\left|\theta \wedge \partial \rho \wedge \bar{\partial} \rho \wedge \omega_{1} \wedge \bar{\omega}_{1}\right| \leq \\
\leq & C \int_{\Omega}(-\rho)|\theta| .
\end{aligned}
$$

Il est clair que le résultat que nous montrons dans ce travail est meilleur que celui qui fait intervenir la pseudo-distance de [5] et [6]. Nous verrons au dernier paragraphe que, en général, il est strictement meilleur. 


\section{Remarque 2.}

Il est naturel de se demander quel résultat on peut obtenir pour les coefficients d'un courant positif fermé de bidegré $(2,2)$. En fait, il est assez facile de se convaincre que dans ce cas, l'inégalité

$$
\int_{\Omega \cap B\left(z_{0}, r\right)} \frac{\left[R_{M}(\delta(z))\right]^{2}}{\delta(z)}|\theta \wedge \partial \rho \wedge \bar{\partial} \rho| \leq C \int_{\Omega}(-\rho)|\theta|
$$

est. satisfaite et que c'cst la condition naturelle.

\section{Schema de la démonstration}

Dans toute la suite, le symbole $A \lesssim B$ signifiera qu'il existe une constante ne dépendant que de $\rho, r, M$ ct de la base $\mathcal{B}$ tel que $A \leq C B$.

Remarquons que $m^{\prime} \geq m$ entraîne $S\left(\mathcal{B}, n^{\prime}\right) \lesssim S(\mathcal{B}, m)$. Par suite, et par raison de symétrie, le théorème se réduit à montrer l'inégalité suivante:

$$
\begin{aligned}
I & =I^{1}+I^{2}= \\
& =\int_{\Omega \cap B\left(z_{0}, r\right)} \frac{\left|\theta \wedge \partial \rho \wedge \bar{\partial} \rho \wedge \omega_{2} \wedge \bar{\omega}_{2}\right|+\left|\theta \wedge \partial \rho \wedge \bar{\partial} \rho \wedge \omega_{1} \wedge \bar{\omega}_{1}\right|}{S(\mathcal{B}, m)}+ \\
& +\int_{\Omega \cap B\left(z_{0}, r\right)} \frac{\left|\theta \wedge \partial \rho \wedge \bar{\partial} \rho \wedge \omega_{1} \wedge \bar{\omega}_{1}\right|}{S\left(L_{2}, \mathcal{B}, m, M\right)} \lesssim \\
& \lesssim \int_{\Omega}(-\rho)|\theta| .
\end{aligned}
$$

Pour simplifier les notations, dans la suite nous noterons $S_{1}=S(\mathcal{B}, m)$ et $S_{2}=S\left(L_{2}, \mathcal{B}, m, M\right)$.

Comme dans [2], la démonstration de cette inégalité est basée sur l'application de la formule de Stokes aux formes

$$
(-\rho) \frac{\theta \wedge \bar{\partial} \rho \wedge \omega_{i} \wedge \tilde{\omega}_{i}}{S_{j}}
$$

Pour ce faire, il est nécéssaire d'une part que le courant $\theta$ soit régulier, et, d'autre part, que les fonctions poids $S_{j}$ soient globalement définies.

Nous supposerons donc tout d'abord que $\theta$ est une forme à cocfficients $\mathcal{C}^{\infty}$ positive et fermée, et démontrerons le résultat sous cette hypothèse. Le cas général s'en déduira aisément avec une régularisation.

Dans le premier sous-paragraphe de la partie IV, nous globalisons les poids en les modifiant à l'extérieur d'unc boule centrée en $z_{0}$. Nous 
obtenons ainsi de nouveaux poids $\mathfrak{S}_{1}$ et $\mathfrak{S}_{2}$ et donnons une formulation équivalente (**) de (*) avec ces nouveaux poids.

En appliquant la formule de Stokes, les dérivées normales et tangentes des poids apparaissent. Le deuxième sous-paragraphe, long et technique, est consacré à établir diverses relations et estimations entre les poids $\mathfrak{S}_{i}$ et leurs dérivées, en particulier des inégalités du type $(-\rho)\left|L_{i} \mathfrak{S}_{j}\right|^{2} \leqslant$ $\mathfrak{S}_{j}^{2+1 / n},(-\rho)\left|N \mathfrak{S}_{j}\right| \lesssim f(\rho) \mathfrak{S}_{j}$ et $(-\rho)|\gamma|^{2} \mathfrak{S}_{1}^{1 / n} \leqq \mathfrak{S}_{2}^{2 / n}$.

C'est dans le troisième sous-paragraphe que, après avoir appliqué la formule de Stokes, nous démontrons la formule $(* *)$. Nous majorons $I^{1}+I^{2}$ par une somme d'intégrales $\sum_{k} I_{k}$ que nous majorons ensuite séparément en utilisant les estimations établies au sous-paragraphe précédent, l'inégalité de Cauchy-Schwarz et la condition de Malliavin (2). Nous montrons que $I_{k} \leq c_{k} I+C \int_{\Omega}(-\rho)|\theta|$, le coefficient $c_{k}$ dépendant d'un paramètre. Pour conclure, il suffit de montrer que l'on peut choisir le paramètre de telle sorte que $\sum_{k} c_{k}<1$.

Remarque sur la composition des sommes $\mathfrak{S}_{1}$ et $\mathfrak{S}_{2}$ consțruites au sous-paragraphe suivant.

Pour $\mathfrak{G}_{1}$ nous avons simplement pris le poids correspondant à la pseudodistance de A. Nagel, E. Stein et $\mathrm{S}$. Wainger, et nous avons essayé de rendre $\mathfrak{S}_{2}$ aussi petit que possible. En oubliant toute question relative à la localisation (fonctions $\psi$ et $\chi_{i}$ ), la composition de $\mathfrak{S}_{2}$ peut s'expliquer, par une analyse rapide de la preuve qui suit, de la manière suivante. Tout d'abord, on remarque que l'on doit avoir $\left.\mathfrak{S}_{2} \gtrsim \mid \partial \rho\left(\mid L_{2}, \bar{L}_{2}\right]\right) \mid$ (proposition 1; cela provient des termes considérés en $b_{1}$ dans la majoration (7) du sous-paragraphe 3). Ensuite, on s'apperçoit que $\mathfrak{S}_{2}$ doit, dans un certain sers, être stable par dérivations dans les directions $N$ et $L_{2}$ (c'est ce que disent précisément la proposition 3 et le (iii) de la proposition 4 ; cette stabilitée sert à contrôler les termes considérés en $d_{1}$ et $\mathrm{d}_{2}$ dans la majoration (9) du sous-paragraphe 3). Cela implique que $\mathfrak{S}_{2}$ doit contenir la partie correspondant à $l_{1}=0$ de $\mathfrak{S}_{2}^{2}$. Par ailleurs, il se produit un phénomène d'échange entre $\mathfrak{S}_{1}$ et $\mathfrak{S}_{2}$ lorsque l'on dérive la forme $\omega_{1} \wedge \bar{\omega}_{1}$. C'est ce phénomène qui implique l'introduction du coefficient $\gamma$ de $L_{1}$ dans la décomposition du crochet $\left[L_{2}, \bar{L}_{2}\right]$ sur la base $\left(N, \bar{N}, L_{1}, \bar{L}_{1}, L_{2}, \bar{L}_{2}\right)$, l'échange étant alors contrôlé par la proposition 2 (précisément, ceci provient des termes $c_{3}$ dans la majoration (8) du sous-paragraphe 3). Alors, en combinant le phénomène d'échange et la stabilitée par rapport aux dérivations en $L_{2}$, on voit facilement que $\mathfrak{S}_{2}$ doit contenir les sommes $\mathfrak{S}_{2}^{\mathbb{4}}$ et $\mathfrak{S}_{2}^{43}$. A ce niveau, si on dérive un des termes de $\mathfrak{S}_{2}$ dans la direction $L_{2}$, il n'apparait pas d'expression d'un type nouveau. Mais comme il faut arreter la somme $\mathfrak{S}_{2}$ à un certain ordre, il 
arrive que des dérivées en $L_{2}$ de certains termes de $\mathcal{S}_{2}$, ainsi construit, ne soient pas dans $\mathfrak{S}_{2}$. Comme la proposition 4 doit, ettre satisfaite, il faut alors rajouter des termes à $\mathfrak{S}_{2}$. C'est ainsi qu'apparaissent. $\mathfrak{S}_{2}^{0}$ et le reste de $\mathfrak{S}_{2}^{2}$ avcc, en particulicr, l'exposant $n-\frac{2 n}{c}$ dans la puissance de $(-\rho)$ pour les termes de $\mathfrak{S}_{2}^{2}$.

\section{Démonstration de la formule $(*)$}

\section{Globalisation et modification des poids $S_{1}$ et $S_{2}$.}

Les champs $L_{i}$ et les formes $\omega_{i}$ n'étant définis que localement dans un voisinage de $z_{0}$, afin de pouvoir appliquer la formule de Stokes au domaine $\Omega$ tout entier, nous élininons ce qui se passe à l'extérieur d'une boule $B\left(z_{0}, r\right)$ en multipliant par une fonction $\psi$ positive, $\mathcal{C}^{\infty}$ dans $\mathbb{C}^{3}$, de support inclus dans $B\left(z_{0}, r\right)$ et identiquement égale à 1 dars la boule $B\left(z_{0}, 2 r / 3\right)$ ( $r$ est choisi de telle sorte que les champs $L_{i}$ soient définis dans $B\left(z_{0}, r\right)$ et que $|\rho| \leq 1$ dans cette boule).

Si on applique la formule de Stokes aux formes

$$
(-\rho) \psi \frac{\theta \wedge \bar{\partial} \rho \wedge \omega_{i} \wedge \bar{\omega}_{i}}{S_{j}}
$$

il apparaît des intégrales de la forme

$$
\int_{\Omega \cap B\left(z_{0}, r\right)} \partial \psi(-\rho) \frac{\theta \wedge \bar{\partial} \rho \wedge \omega_{i} \wedge \bar{\omega}_{i}}{S_{j}}
$$

Ces intégrales seront majorées trivialement, ce qui ne peut se faire que si $S_{j} \gtrsim 1$ lorsque $\partial \psi \neq 0$, et ce n'est pas à priori le cas. Nous allons donc modifier les fonctions $S_{j}$ en introduisant deux nouvelles fonctions $\chi_{1}$ et. $\chi_{2}$ positives, $\mathcal{C}^{\infty}$ dans $\mathbb{C}^{3}$, telles que $\chi_{1}+\chi_{2} \equiv 1, \chi_{1} \equiv 1$ darns $B\left(z_{0}, r / 2\right)$ et $\chi_{2} \equiv 1$ dans $\mathbb{C}^{3} \backslash B\left(z_{0}, 2 r / 3\right)$.

La base $\mathcal{B}, r$, les fonctions $\psi, \chi_{1}$ et $\chi_{2}$ étant fixées, nous pouvons maintenant défnir de nouvelles fonctions $\mathfrak{S}_{1}$ et $\mathfrak{S}_{2}$ de la manière suivante:

Pour $\mathcal{L}=\left(L^{1}, \ldots, L^{k}\right), k \geq 2$, posons

$$
\begin{aligned}
& \mathcal{L}(\partial \rho)=L^{1} \ldots L^{k-2}\left(\chi_{1} \partial \rho\left(\left[L^{k-1}, L^{k}\right]\right)\right), \\
& \mathcal{L}\left(\chi_{2}\right)=L^{1} \ldots L^{k-2}\left(\chi_{2}\right)
\end{aligned}
$$

et, de plus, si $\gamma$ est le coefficient de $L_{1}$ dans la décomposition de $\left[L_{2}, \bar{L}_{2}\right]$ dans la base $\left(N, \tilde{N}, L_{1}, \bar{L}_{1}, L_{2}, \bar{L}_{2}\right)$, pour $\tilde{\mathcal{L}} \in W_{1}$,

$$
\tilde{\mathcal{L}}(\gamma)=L^{1} \ldots L^{k-2}\left(\chi_{1} \gamma\right)
$$


étant entendu que dans ces formules, si $k=2$, on a $\mathcal{L}(\partial \rho)=\chi_{1} \partial \rho\left(\left[L^{1}, L^{2}\right]\right)$, $\mathcal{L}\left(\chi_{2}\right)=\chi_{2}$ et $\widetilde{\mathcal{L}}\left(\chi_{1} \gamma\right)=\chi_{1} \gamma$.

Au cours de la démonstration, nous serrons amenés à dériver terme à terme les cocfficients de $S_{j}$. Pour faciliter leurs majorations il est utile de n'avoir à considérer que des puissances supérieures à 1 . Pour cela, chacun des termes sera élevé à une puissance $n$ fixée, $n>M$.

Nous pouvons maintenant définir les nouvelles fonctions $\mathfrak{S}_{j}$ : tout, d'abord $\mathcal{S}_{1}$ en posant

$$
\mathfrak{S}_{1}=\sum_{\mathcal{L} \in W_{1}}\left(|\mathcal{L}(\partial \rho)|^{\frac{2 n}{\hbar}}+\left|\mathcal{L}\left(\chi_{2}\right)\right|^{\frac{2 n}{\hbar}}\right)(-\rho)^{n-\frac{2 n}{k}}+(-\rho)^{n-\frac{2 n}{m}} .
$$

Définissons cnsuite $\mathfrak{S}_{2}$ par

$$
\mathfrak{S}_{2}=\mathfrak{S}_{2}^{0}+\mathfrak{S}_{2}^{2}+\mathfrak{S}_{2}^{4}+\mathfrak{S}_{2}^{43}+(-\rho)^{n-\frac{2 n}{M}},
$$

où l'on a noté successivement

$$
\begin{aligned}
& S_{2}^{0}=\sum_{\mathcal{L} \in W_{0}}\left(|\mathcal{L}(\partial \rho)|^{\frac{2 n}{k}}+\left|\mathcal{L}\left(\chi_{2}\right)\right|^{\frac{2 n}{k}}\right)(-\rho)^{n-\frac{2 n}{k}+\frac{2 n}{m}-\frac{2 n}{M}}, \\
& \mathfrak{S}_{2}^{2}=\sum_{\mathcal{L} \in \mathbb{W}_{2}}\left(|\mathcal{L}(\partial \rho)|^{\frac{2 n}{\tau_{2}}}+\left|\mathcal{L}\left(\chi_{2}\right)\right|^{\frac{2 n}{\gamma_{2}}}\right)(-\rho)^{n-\frac{2 n}{\kappa}}, \\
& \mathcal{S}_{2}^{A}=\sum_{\tilde{\mathcal{L}} \in W_{4}}|\tilde{\mathcal{L}}(\gamma)|^{\frac{2 n}{i_{2}}}(-\rho)^{n-\frac{2 n}{m \tilde{i}_{2}}},
\end{aligned}
$$

et,

$$
\mathcal{S}_{2}^{43}=\sum_{\tilde{\mathcal{L}} \in W_{4}} \sum_{\mathcal{L} \in W_{3}}|\tilde{\mathcal{L}}(\gamma)|^{\frac{2 n i_{1}}{D}}\left(|\mathcal{L}(\partial \rho)|^{\frac{2 n}{D}}+\left|\mathcal{L}\left(\chi_{2}\right)\right|^{\frac{2 n}{D}}\right)(-\rho)^{n-\frac{2 n}{D}}
$$

où $D=l_{1} \bar{l}_{2}+l_{2}$.

Remarquons que, dans la boule $B\left(z_{0}, r / 2\right)$, on a $\chi_{1} \equiv 1, \chi_{2} \equiv 0$ et $\psi \equiv 1$, et par suite, $S_{j} \simeq \mathfrak{S}_{j}^{1 / n}$. Pour démontrer l'inégalité $(*)$, il suffit donc de montrer que

$$
\begin{aligned}
& \int_{\Omega} \psi \frac{i^{2} \theta \wedge \partial \rho \wedge \bar{\partial} \rho \wedge\left(\omega_{1} \wedge \bar{\omega}_{1}+\omega_{2} \wedge \bar{\omega}_{2}\right)}{\mathfrak{S}_{1}^{1 / n}}+ \\
+ & \int_{\Omega} \psi \frac{i^{2} \theta \wedge \partial \rho \wedge \bar{\partial} \rho \wedge \omega_{1} \wedge \bar{\omega}_{1}}{\mathfrak{S}_{2}^{1 / n}} \lesssim \\
\lesssim & \int_{\Omega}(-\rho)|\theta| .
\end{aligned}
$$




\section{Quelques propriétês des fonctions $\mathfrak{S}_{i}$.}

\section{Proposition 1.}

1) Dans $B\left(z_{0}, r\right)$, on $a$

$$
\mathfrak{S}_{1}^{1 / n} \gtrsim\left|\partial \rho\left(\left[L_{1}, \bar{L}_{1}\right]\right)\right|+\left|\partial \rho\left(\left[L_{2}, \bar{L}_{2}\right]\right)\right| .
$$

et

$$
\mathfrak{S}_{2}^{1 / n} \geq\left|\partial \rho\left(\left[L_{2}, \bar{L}_{2}\right]\right)\right| .
$$

2) Dans $B\left(z_{0}, r\right) \backslash B\left(z_{0}, 2 r / 3\right)$, on $a \mathfrak{S}_{1} \geq 1$ et $\mathfrak{S}_{2} \geq 1$.

Démonstration: Considérons tout d'abord le cas de $\mathfrak{S}_{1}$. Si $m=2$, on a $\mathfrak{S}_{1}=1$, et les deux propriétés sont trivialement vérifiées. Supposons $m>2$. Alors, $\mathcal{L}=\left(L_{i}, \bar{L}_{i}\right) \in W_{1}$ et

$$
\mathfrak{S}_{1} \geq\left|\chi_{1} \partial \rho\left(\left[L_{1} \bar{L}_{1}\right]\right)\right|^{n}+\chi_{2}^{n}+\left|\chi_{1} \partial \rho\left(\left[L_{2}, \bar{L}_{2}\right]\right)\right|^{n}+\chi_{2}^{n} .
$$

$\mathrm{Si}\left|\partial \rho\left(\left[L_{i} \bar{L}_{i}\right]\right)\right| \geq 1$, on a $\left|\chi_{1} \partial \rho\left(\left[L_{i}, \bar{L}_{i}\right]\right)\right|+\chi_{2} \geq 1$, ct si $\left|\partial \rho\left(\left[L_{i}, \bar{L}_{i}\right]\right)\right|<1$, on a $\left|\chi_{1} \partial \rho\left(\left[L_{i} \bar{L}_{i}\right]\right)\right|+\chi_{2} \geq\left|\partial \rho\left(\left\{L_{i} \bar{L}_{i}\right\}\right)\right|$, ce qui démontre, pour $\mathfrak{S}_{1}$, lat première proprićtć.

De plus, à l'extérieur de la boule $B\left(z_{0}, 2 r / 3\right), \chi_{2} \equiv 1$, et la seconde propriété est vraie pour $\mathfrak{G}_{1}$.

De la même, manière, si $M=2, \mathfrak{S}_{2}=1$, et si $M>2, \mathcal{L}=\left(L_{2}, \bar{L}_{2}\right) \in$ $W_{2}, \mathfrak{S}_{2} \geq\left|\chi_{1} \partial \rho\left(\left(L_{2}, \bar{L}_{2}\right]\right)\right|^{n}+\chi_{2}^{n}, \operatorname{car} c(\mathcal{L})=2$, ct nous avons les mèmes propriétés.

\section{Lemme 1.}

Dans la boule $B\left(z_{0}, r\right)$, on peut, majorer $\mathfrak{S}_{1}$ à une constante près par

$$
\begin{aligned}
T_{1} & =\sum_{\mathcal{L} \in W_{0}}\left(|\mathcal{L}(\partial \rho)|^{\frac{2 n}{T_{1}}}+\left|\mathcal{L}\left(\chi_{2}\right)\right|^{\frac{2 n}{T_{1}}}\right)(-\rho)^{n-\frac{2 n}{T_{1}}}+ \\
& +\sum_{\mathcal{L} \in W_{2}}\left(|\mathcal{L}(\partial \rho)|^{\frac{2 n}{\tau_{2}}}+\left|\mathcal{L}\left(\chi_{2}\right)\right|^{\frac{2 n}{T_{2}}}\right)(-\rho)^{n-\frac{2 n}{c}}+(-\rho)^{n-\frac{2 n}{m}}
\end{aligned}
$$

Démonstration: En effet, soit $\mathcal{L} \in W_{1}$, et posons

$$
A=\left(|\mathcal{L}(\partial \rho)|^{\frac{2 n}{k}}+\left|\mathcal{L}\left(\chi_{2}\right)\right|^{\frac{2 n}{k}}\right)(-\rho)^{\gamma_{2}-\frac{2 n}{k}} .
$$

Alors, ou bien $\mathcal{L} \in W_{0}$ et comme $l_{2}(\mathcal{L})=k, A$ se trouve dans la première somme de $T_{1}$, ou bien $\mathcal{L} \in W_{2}$ et

$$
A \simeq\left[\left(\mathcal{L}(\partial \rho)^{\frac{2 n}{i_{2}}}+\left|\mathcal{L}\left(\chi_{2}\right)\right|^{\frac{2 n}{\tau_{2}}}\right)(-\rho)^{n-\frac{2 n}{c}}\right]^{\frac{i_{2}}{k}}(-\rho)^{\frac{n-\frac{2 n}{k}}{k}-\frac{i_{2} n}{k}+2 \frac{i_{2} n}{c k}} .
$$


Or,

$$
\frac{l_{2}}{c}=\frac{m-l_{1}}{m}, 1-\frac{2}{k}-\frac{l_{2}}{k}+\frac{2 l_{2}}{c k}=1-\frac{l_{2}}{k}-\frac{2 l_{1}}{k m}=\frac{l_{1}}{k}\left(1-\frac{2}{m}\right)
$$

d'où:

$$
(-\rho)^{n\left(1-\frac{2}{k}-\frac{t_{2}}{k}+\frac{2 l_{2}}{c k}\right)}=(-\rho)^{\frac{n l_{1}}{k}\left(1-\frac{2}{m}\right)} \leq T_{1}^{\frac{t_{1}}{k}}
$$

et done $A \leq T_{1}$, ce qui achève la démonstration du lemme.

\section{Proposition 2.}

Dans la boule $B\left(z_{0}, r\right)$ les fonctions $\mathfrak{S}_{1}$ et $\mathfrak{S}_{2}$ vérifient l'inégalité suivante:

$$
(-\rho)|\gamma|^{2} \mathfrak{S}_{1}^{1 / n} \leqslant \mathfrak{G}_{2}^{2 / \pi^{2}}
$$

Dćmonstration: D'après le lemme précédent, il suffit de montrer que $(-\rho)|\gamma|^{2} T_{1}^{1 / \pi} \lesssim \mathfrak{S}_{2}^{2 / n}$. Nous allons vérifier que, pour chaque composante $X$ de $T_{1}$, on at

$$
(-\rho)|\gamma|^{2} X^{1 / n} \leqq \mathcal{G}_{2}^{2 / n}
$$

a) Considérons tout d'abord $X=\left(|\mathcal{L}(\partial \rho)|^{\frac{2 n}{1_{2}}}+\left|\mathcal{L}\left(\chi_{2}\right)\right|^{\frac{2 n}{\gamma_{2}}}\right)(-\rho)^{n-\frac{2 n}{c}}$, pour $\mathcal{L} \in W_{2}$.

Ce terme apparaît aussi dans $\mathfrak{S}_{2}$ et le résultat est trivial car $(-\rho) \lesssim$ $\mathfrak{S}_{2}^{1 / n}$.

b) Considérons ensuitc $X=(-\rho)^{n(1-2 / m)}$. Si $M \leq 2 m$, on a

$$
(-\rho)|\gamma|^{2} X^{1 / n} \leqslant(-\rho)^{2-2 / m} \leqslant\left((-\rho)^{1-2 / M}\right)^{2} \leqslant \mathbb{S}_{2}^{2 / n}
$$

Si $M>2 n, \tilde{\mathcal{L}}=\left(L_{2}, L_{2}\right) \in W_{4}$, et, $|\gamma|^{n}(-\rho)^{n-n / m}$ est une composante de $\mathfrak{S}_{2}$, et. par suite, $(-\rho)|\gamma|^{2} X^{1 / n}=|\gamma|^{2}(-\rho)^{2-2 / m} \leq \mathfrak{S}_{2}^{2 / n}$.

c) Considérons enfin les termes $X=\left(|\mathcal{L}(\partial \rho)|^{\frac{2 n}{k}}+\left|\mathcal{L}\left(\chi_{2}\right)\right|^{\frac{2 n}{k}}\right)(-\rho)^{n-\frac{2 n}{k}}$, pour $\mathcal{L} \in W_{0}$ (ce qui implique $m>2$ ).

Si $M \leq 2 m$, on a

$$
\begin{aligned}
& (-\rho)|\gamma|^{2} X^{1 / n} \leqq\left(|\mathcal{L}(\partial \rho)|^{\frac{2}{5}}+\left|\mathcal{L}\left(\chi_{2}\right)\right|^{\frac{2}{4}}\right) \\
& (-\rho)^{1-\frac{2}{k}+\frac{2}{m}-\frac{2}{M}}(-\rho)^{1-\frac{2}{M}}(-\rho)^{\frac{4}{M}-\frac{2}{M}} \lesssim \mathfrak{G}_{2}^{2 / n},
\end{aligned}
$$

$\operatorname{car} \frac{4}{M}-\frac{2}{M} \geq 0$. 
Si $M>2 m, \tilde{\mathcal{L}}=\left(L_{2}, L_{2}\right) \in W_{4}$, et, comme $\mathcal{L} \in W_{3}\left(W_{0} \subset W_{3}\right)$, $|\gamma|^{n}\left(|\mathcal{L}(\partial \rho)|^{n / k}+\left|\mathcal{L}\left(\chi_{2}\right)\right|^{n / k}\right)(-\rho)^{n-n / k}$ est une composante de $\mathfrak{S}_{2}$, et $\mathbb{S}_{2}^{2 / n}$ majore $(-\rho)|\gamma|^{2} X^{1 / n}$.

Nous allons mairitenant donner deux propositions qui étudient, le comportement des dérivées des fonctions $\mathfrak{S}_{1}$ et $\mathfrak{S}_{2}$ par rapport aux champs tangentiels et normal.

\section{Proposition 3.}

Il existe deux constantes $c_{0}$ et $C, 0<c_{0}<1, C$ ne dépendant que de $\rho, r$, de la base $\mathcal{B}=\left(L_{1}, L_{2}\right)$ et des fonctions $\chi_{1}$ et $\chi_{2}$, telles que, dans la boule $B\left(z_{0}, r\right)$, on a

$$
\begin{aligned}
& \frac{1}{n}(-\rho)\left|N\left(\mathfrak{S}_{1}\right)\right| \leq\left(c_{0}+C(-\rho)^{\frac{2}{m}}\right) \mathfrak{S}_{1}, \\
& \frac{1}{n}(-\rho)\left|N\left(\mathfrak{S}_{2}\right)\right| \leq\left(c_{0}+C(-\rho)^{\frac{2}{M}}\right) \mathfrak{S}_{2},
\end{aligned}
$$

Démonstration: $\mathfrak{S}_{i}$ étant une somme de termes de la formc $X^{\alpha}(-\rho)^{\beta}$, où $\alpha>1$, nous allons montrer que, pour chacun d'entre eux

$$
\begin{aligned}
(-\rho)\left|N\left(X^{\alpha}(-\rho)^{\beta}\right)\right| \leq(1- & \left.s_{i}\right) n X^{\alpha}(-\rho)^{\beta}+ \\
& +C(-\rho)^{s_{i}} \mathfrak{G}_{i} \text {, où } s_{1}=\frac{2}{m} \text { et } s_{2}=\frac{2}{M} .
\end{aligned}
$$

Les inćgalités cherchées seront alors obtenues en choisissant $c_{0}=\left(1-\frac{2}{M}\right)$.

En effet, puisque nous avons choisi $N(-\rho) \equiv 1$ et que les termes $X^{\alpha}(-\rho)^{\beta}$ sont tels que, dans $\mathfrak{S}_{1}, \beta \leq n\left(1-\frac{2}{M}\right)$ et dans $\mathfrak{G}_{2}, \beta \leq n\left(1-\frac{2}{M}\right)$, on a

$$
(-\rho)\left|N\left(X^{\alpha}(-\rho)^{\beta}\right)\right| \leq \alpha X^{\alpha-1}|N(X)|(-\rho)^{\beta+1}+\left(1-s_{i}\right) n X^{\alpha}(-\rho)^{\beta} .
$$

Considérons donc le terme $\alpha X^{\alpha-1}|N(X)|(-\rho)^{\beta+1}$ de l'inćgalité ci dessus. Il est majorć par $C_{1} X^{\alpha-1}(-\rho)^{\beta+1}$ où $C_{1}$ est une constante qui ne dépend que de la base $B, \rho_{1} r$ ct des fonctions $\chi_{1}$ et $\chi_{2}$. Si on écrit

$$
X^{\alpha-1}(-\rho)^{\beta+1}=\left[X^{\alpha}(-\rho)^{\beta}\right]^{1-\frac{1}{\alpha}}(-\rho)^{\frac{\beta+a}{\alpha}},
$$

le terme entre crochets est inférieur à $\mathfrak{S}_{i}$. En utilisant l'inégalité $\mathfrak{S}_{i} \geq$ $(-\rho)^{n\left(1-s_{i}\right)}$, il vient

$$
X^{\alpha-1}(-\rho)^{\beta+1} \leq \mathfrak{S}_{i}^{1-\frac{1}{\alpha}} \mathfrak{S}_{i}^{\frac{1}{\alpha}}(-\rho)^{\frac{\beta+\alpha-n}{\alpha}}(-\rho)^{\frac{n_{s_{i}}}{\alpha}},
$$

et comme $\alpha$ est inférieur à $n$, pour achever la démonstration, il suffit de montrer que (sauf dans le cas $\alpha=0$ qui correspond au terme $(-\rho)^{n\left(1-s_{i}\right)}$ ) on a toujours $\alpha+\beta \geq n$. Or, $\alpha+\beta$ est soit égal à $n$, soit $n+\frac{2}{m}-\frac{2}{M}$, soit à $\frac{2}{l_{2}}-\frac{2}{c}+n$, soit à $n+\frac{2 n}{l_{2}}\left(1-\frac{1}{n}\right)$ (dans le cas des sornmes doubles on considère $X=|\widetilde{\mathcal{L}}(\gamma)|^{l_{1}}|\mathcal{L}(\partial \rho)|$ ou $\left.X=|\widetilde{\mathcal{L}}(\gamma)|^{l_{1}}\left|\mathcal{L}\left(\chi_{2}\right)\right|\right)$, et comme $m \leq M$ et $\frac{t_{2}}{c} \leq 1$, on a bien $\alpha+\beta \geq n$. 


\section{Proposition 4.}

Dans la boule $B\left(z_{0}, r\right)$, on a les inégalités suivantes:

(i) $(-\rho)\left|L_{1} \mathfrak{S}_{1}\right|^{2} \lesssim \mathfrak{S}_{1}^{2+1 / n}$;

(ii) $(-\rho)\left|L_{2} \mathfrak{S}_{1}\right|^{2} \lesssim \mathfrak{S}_{1}^{2+1 / n}$

(iii) $(-\rho)\left|L_{2} \mathfrak{S}_{2}\right|^{2} \lesssim \mathfrak{S}_{2}^{2+1 / n}$

Pour vérifier ces inćgalités, établissons tout d'abord deux lemmes:

\section{Lemme 2.}

Soient $\alpha, \beta, A, B$, et $N$ des réels positifs vérifiant na $>1$, $\frac{2}{\alpha}-\frac{2}{A}+1 \geq 0$ et $\frac{B}{A}-\frac{\beta}{\alpha} \geq-\frac{1}{N}\left(\frac{2}{\alpha}-\frac{2}{A}+1\right)$. Soit $f$ une fonction positive telle que $f^{n \alpha}$ soit de classe $\mathcal{C}^{1}$. Posons $F=f^{n \alpha}(-\rho)^{n(1-\beta)}$. Alors pour tout champ tangent $L$, en tout point où $(-\rho) \leq 1$, on $a$ :

$$
(-\rho)|L F|^{2} \leq n^{2} \alpha^{2}\left[F+|L f|^{n A}(-\rho)^{n(1-B)}+(-\rho)^{n(1-2 / N)}\right]^{2+1 / n} .
$$

Démonstration: $|L F|^{2}=\left|n \alpha f^{n \alpha-1} L f(-\rho)^{n(1-\beta)}\right|^{2}$. Comme

$$
\left(f^{n \alpha-1}\right)^{2}=\left[f^{n \alpha}(-\rho)^{n(1-\beta)}\right]^{2-\frac{2}{\pi \alpha}}(-\rho)^{\frac{2}{\alpha}-\frac{2 \beta}{\alpha}-2 n(1-\beta)},
$$

et,

$$
|L f|^{2}=\left[|L f|^{n A}(-\rho)^{n(1-B)}\right]^{\frac{2}{n A}}(-\rho)^{\frac{2 B}{A}-\frac{2}{A}},
$$

on a donc, en posart $G=F+|L f|^{n A}(-\rho)^{n(1-B)}+(-\rho)^{n(1-2 / N)}$,

$$
|L F|^{2} \leq n^{2} \alpha^{2} G^{2-\frac{2}{n \alpha}+\frac{2}{n \alpha}}(-\rho)^{\frac{2}{\alpha}-\frac{2}{\lambda}+\frac{2 \pi}{A}-\frac{2 \beta}{\alpha}} .
$$

Comme $\frac{2}{\alpha}-\frac{2}{A}+1 \geq 0$, on a $G^{\frac{1}{n}\left(\frac{2}{\alpha}-\frac{2}{\lambda}+1\right)} \geq(-\rho)^{\left(\frac{2}{\alpha}-\frac{2}{\lambda}+1\right)\left(1-\frac{2}{N}\right)}$, ce qui donne l'inégalité cherchée dès que

$$
1+\frac{2}{\alpha}-\frac{2}{A}+\frac{2 B}{A}-\frac{2 \beta}{\alpha} \geq\left(\frac{2}{\alpha}-\frac{2}{A}+1\right)\left(1-\frac{2}{N}\right),
$$

c'est-à-dire,

$$
\frac{B}{A}-\frac{\beta}{\alpha} \geq-\frac{1}{N}\left(\frac{2}{\alpha}-\frac{2}{A}+1\right)
$$

ce qui est la condition supposée. 


\section{Lemme 3.}

Soient $\alpha, \beta, A, B, A^{\prime}$ et $B^{\prime}$ des réels positifs vérifiant les relations $n \alpha>1, n \beta>1, \frac{2}{\alpha}-\frac{2}{A}+1=\frac{2}{\beta}-\frac{2}{\beta^{\prime}}+1=0$ et $\frac{\beta}{\alpha}=\frac{B}{A}=\frac{B^{\prime}}{A^{\prime}}$. Soient $f$ et $g$ deux fonctions positives telles que $f^{n \alpha}$ et $g^{n \beta}$ soient de classe $\mathcal{C}^{1}$. Posons $F=f^{n \alpha} g^{n \beta}(-\rho)^{n(1-\beta)}$. Alors pour tout champ tangent $L$, en tout point où $(-\rho) \leq 1$, on $a$ :

$$
\begin{aligned}
& (-\rho)|L F|^{2} \leq 2 n^{2}\left(\alpha^{2}+\beta^{2}\right) \times \\
& \quad \times\left[F+|L f|^{n A} g^{n B}(-\rho)^{n(1-B)}+f^{n A^{\prime}}|L g|^{n B^{\prime}}(-\rho)^{n\left(1-B^{\prime}\right)}\right]^{2+\frac{1}{n}} .
\end{aligned}
$$

Démonstration: On a

$$
\begin{aligned}
(-\rho)|L F|^{2} & \leq n^{2} \alpha^{2} f^{2 n \alpha-2} g^{2 n \beta}(-\rho)^{2 n(1-\beta)+1}|L f|^{2}+ \\
& +n^{2} \beta^{2} f^{2 n \alpha} g^{2 n \beta-2}(-\rho)^{2 n(1-\beta)+1}|L g|^{2} .
\end{aligned}
$$

Le résultat est donc trivial si l'une des deux fonctions $f$ ou $g$ s'annule. Supposons donc qu'elles ne s'annulent pas. On a alors d'une part,

$$
\begin{aligned}
g^{2 n \beta} f^{2 n \alpha-2} & =\left(f^{n \alpha} g^{n \beta}(-\rho)^{n(1-\beta)}\right)^{2-\frac{2}{n \alpha}} g^{\frac{2 \beta}{\alpha}}(-\rho)^{-2 n(1-\beta)+\frac{2}{a}-\frac{2 \beta}{\alpha}} \\
|L f|^{2} & =\left(|L f|^{n A} g^{n B}(-\rho)^{n(1-B)}\right)^{\frac{2}{n A}} g^{-\frac{2 \beta}{\lambda}}(-\rho)^{-\frac{2}{\lambda}+\frac{2 B}{\lambda}}
\end{aligned}
$$

ce qui donne

$$
\begin{gathered}
f^{2 n \alpha-2} g^{2 n \beta}(-\rho)^{2 n(1-\beta)+1}|L f|^{2}=F^{2-\frac{2}{n \alpha}}\left[|L f|^{n A} g^{n B}(-\rho)^{n(1-B)}\right]^{\frac{2}{n \lambda}}, \\
\text { car } \frac{2}{\alpha}-\frac{2}{A}+1=0 \text { et } \frac{2 B}{A}-\frac{2 \beta}{\alpha}=0, \text { et, d'autre part, } \\
f^{2 n \alpha} g^{2 n \beta-2}=\left(f^{n \alpha} g^{n \beta}(-\rho)^{n(1-\beta)}\right)^{2-\frac{2}{n \beta}} f^{\frac{2 \alpha}{\beta}}(-\rho)^{-2 n(1-\beta)+\frac{2}{\beta}-2}, \\
|L g|^{2}=\left(f^{n A^{\prime}}|L g|^{n B^{\prime}}(-\rho)^{n\left(1-B^{\prime}\right)}\right)^{\frac{2}{n B^{\prime}}} f^{-\frac{2 A^{\prime}}{B^{\prime}}}(-\rho)^{2-\frac{2}{B^{\prime}}},
\end{gathered}
$$

ce qui en utilisant les relations $\frac{2}{\beta}-\frac{2}{B^{\prime}}+1=0$ et $\frac{\alpha}{\beta}=\frac{\Lambda^{\prime}}{B^{\prime}}$ donne à son tour

$$
f^{2 n \alpha} g^{2 n \beta-2}|L g|^{2}(-\rho)^{2 n(1-\beta)+1}=F^{2-\frac{2}{n \beta}}\left[f^{n A^{\prime}}|L g|^{n B^{\prime}}(-\rho)^{n\left(1-B^{\prime}\right)}\right]^{\frac{2}{n B^{\prime}}} .
$$

Ceci achève la démonstration du lemrne $3 \operatorname{car} \frac{2}{n A}-\frac{2}{n \alpha}=\frac{2}{n B^{\prime}}-\frac{2}{n \beta}=\frac{1}{n}$. 
Démonstration de la proposition 4:

Par définition, les fonctions $\mathfrak{S}_{1}$ et $\mathfrak{S}_{2}$ sont des sommes de termes de la forme

$$
|\widetilde{\mathcal{L}}(\gamma)|^{a}|\mathcal{L}(\partial \rho)|^{b}(-\rho)^{c} \text { et }|\widetilde{\mathcal{L}}(\gamma)|^{a}\left|\mathcal{L}\left(\chi_{2}\right)\right|^{b}(-\rho)^{c} .
$$

Nous montrerons done que, pour chacun des termes $X$ composant la somme $\mathfrak{S}_{1}$ et chacun des termes $Y$ composant lia somme $\mathfrak{S}_{2}$, on a $(-\rho)\left|L_{1} X\right|^{2} \lesssim \mathfrak{S}_{1}^{2+1 / n},(-\rho)\left|L_{2} X\right|^{2} \lesssim \mathfrak{S}_{1}^{2+1 / n}$ et $(-\rho)\left|L_{2} Y\right|^{2} \leqslant \mathfrak{S}_{2}^{2+1 / n}$.

Pour cela, nous utiliserons, suivant la forme de $X$ ou de $Y$, le lemme 2 ou le lemme 3. Les majorants obtenus sont de la forme (somme de trois termes) ) $^{2+1 / n}$. Le premier de ces termes est $X$ ou $Y$ lui même (donc $\leq \mathfrak{S}_{i}$ ) et, dans les trois premiers cas, le dernier est, une puissance de $(-\rho)$ intervenant dans $\mathfrak{S}_{i}$ (donc $\leq \mathfrak{S}_{i}$ ). Nous montrerons que, suivant les cas, les termes manquants sont, soit une composante de $\mathfrak{S}_{i}$, soit majorés par une puissance adéquate de $(-\rho)$.

\section{Premier cas.}

$$
X=|\mathcal{L}(\partial \rho)|^{2 n / k}(-\rho)^{n(1-2 / k)}
$$

ou

$$
X^{\prime}=\left|\mathcal{L}\left(\chi_{2}\right)\right|^{2 n / k}(-\rho)^{n(1-2 / k)}
$$

pour $\mathcal{L} \in W_{1}$.

Soit $L=L_{1}$ ou $L_{2}$. Appliquons le lemrne 2 avec les paramètres $\alpha=\beta=2 / k, A=B=2 /(k+1)$ et $N=m$. Il vient.

$$
(-\rho)|L X|^{2} \leq\left(X+|L \mathcal{L}(\partial \rho)|^{\frac{2 n}{k+1}}(-\rho)^{n\left(1-\frac{2}{k+1}\right)}+(-\rho)^{n\left(1-\frac{2}{m}\right)}\right)^{2+\frac{1}{n}} .
$$

Examinons le second terme de la parenthèse. Si $k<m-1,(L, \mathcal{L}) \in W_{1}$ et ce terme est un des termes composant la somme $\mathfrak{S}_{1}$. Si $k=m-1$, il est majoré, à une constante près, par $(-\rho)^{n(1-2 / m)}$.

La démonstration pour $X^{\prime}$ est strictement la même que celle pour $X$ en remplaçant $\mathcal{L}(\partial \rho)$ par $\mathcal{L}\left(\chi_{2}\right)$.

Ceci achève la preuve de (i) et (ii). Nous démontrons mintenant (iii).

Deuxième cas. $Y=|\mathcal{L}(\partial \rho)|^{2 n / l_{2}}(-\rho)^{n(1-2 / c)}$, on $Y^{\prime}=\left|\mathcal{L}\left(\chi_{2}\right)\right|^{2 n / l_{2}}$ $(-\rho)^{n(1-2 / c)}$, pour $\mathcal{L} \in W_{2}$.

Appliquons le lemme 2 avec $L=L_{2}$ et avec les paramètres $\alpha=\frac{2}{l_{2}}$, $\beta=\frac{2}{c}=\frac{2}{l_{2}}\left(\frac{m-t_{1}}{m}\right), A=\frac{2}{l_{2}+1}, B=\frac{2}{l_{2}+1}\left(\frac{m-l_{1}}{m}\right)$ et $N=M$. Il vient $(-\rho)\left|L_{2} Y\right|^{2} \leqslant\left(Y+\left|L_{2} \mathcal{L}(\partial \rho)\right|^{\frac{2 n}{l_{2}+1}}(-\rho)^{n\left(1-\frac{2}{t_{2}+1}\left(\frac{m-t_{1}}{\tau^{2}}\right)\right)}+(-\rho)^{n\left(1-\frac{2}{M}\right)}\right)^{2+\frac{1}{n}}$. 
Examinons le sccond terme de la parenthèse. Si $l_{2}<M\left(\frac{m-l_{1}}{m}\right)-1$, $\left(L_{2}, \mathcal{L}\right) \in W_{2}$ ct $c\left(\left(L_{2}, \mathcal{L}\right)\right)=\frac{\left(l_{2}+1\right) m}{m-l_{1}}$, et ce terme est unc composante de $\mathfrak{S}_{2}$. Si $l_{2} \geq M\left(\frac{\pi n-l_{1}}{m}\right)-1$, il est majoré, à une constante près par $(-\rho)^{n(1-2 / M)}$.

La démonstration pour $Y^{\prime}$ est strictement la même que celle pour $Y$.

Troisième cas.

$$
Y=|\mathcal{L}(\partial \rho)|^{\frac{2 n}{T_{1}}}(-\rho)^{n\left(1-\frac{2}{t_{1}}+\frac{2}{m}-\frac{2}{M}\right)},
$$

ou

$$
Y=\left|\mathcal{L}\left(\chi_{2}\right)\right|^{\frac{2 n}{T_{1}}}(-\rho)^{n\left(1-\frac{2}{T_{1}}+\frac{2}{m}-\frac{2}{M}\right)}
$$

pour $\mathcal{L} \in W_{0}$

Appliquons le lemme 2 avec $L=L_{2}$ et avec les paramòtres $\phi=\frac{2}{l_{1}}$, $\beta=\frac{2}{l_{1}}+\frac{2}{M}-\frac{2}{m}, A=2, B=2\left(\frac{m-l_{1}}{m}\right)$ et $N=M$, en remarquant quc $\frac{2}{\alpha}-\frac{2}{A}+1=l_{1}$ et $\frac{B 3}{A}-\frac{\beta}{\alpha}=-\frac{l_{1}}{M}$. II vient

$$
(-\rho)\left|L_{2} Y\right|^{2} \leq\left(Y+\left|L_{2} \mathcal{L}(\partial \rho)\right|^{2 n_{2}}(-\rho)^{n\left(1-2\left(\frac{m t_{1}}{m}\right)\right)}+(-\rho)^{n\left(1-\frac{2}{M}\right)}\right)^{2+\frac{1}{n}} \text {. }
$$

Examinons le deuxième terma de la parenthcso. Si $l_{1}<m\left(1-\frac{1}{M}\right)$ alors $\left(L_{2}, \mathcal{L}\right) \in W_{2}, l_{2}\left(\left(L_{2}, \mathcal{L}\right)\right)=1, c\left(\left(L_{2}, \mathcal{L}\right)\right)=\frac{m_{2}}{\pi_{t}-l_{1}}$, et ce terme est une composante de $\mathfrak{S}_{2}$. Si $l_{1} \geq m\left(1-\frac{1}{M}\right)$, il est majoré, à une constante près, par $(-\rho)^{r(1-2 / M)}$

La démonstration pour $Y^{\prime}$ est strictement la même que pour $Y$.

Quatrìme cas. $Y=|\widetilde{\mathcal{L}}(\gamma)|^{\frac{2 n}{\sqrt{2}}}(-\rho)^{n\left(1-\frac{2}{i_{2 m}}\right)}$, pour $\tilde{\mathcal{L}} \in W_{1}$

Appliquons le: lemme 2 avec $L=L_{2}$ et avec les parametres $\alpha=\frac{2}{i_{2}}$, $\beta=\frac{2}{l_{2} m}, A=\frac{2}{l_{2}+1}, B=\frac{2}{\left(l_{2}+1\right) m}$ et $N=M$. Il vicrit,

$$
(-\rho)\left|L_{2} Y\right|^{2} \leq\left(Y+\mid L_{2} \overrightarrow{\mathcal{L}}(\gamma)^{\frac{2 n}{i_{2}+1}}(-\rho)^{n\left(1-\frac{2}{\left(i_{2}+1\right) m}\right)}+(-\rho)^{n\left(1-\frac{2}{M}\right)}\right)^{2+\frac{1}{h_{1}}}
$$

Examinons le deuxième terme de la parenthèse. Si $\tilde{l}_{2}<\frac{M}{m}-1,\left(L_{2}, C\right) \in$ $W_{1}$ et ce terme est une composante de $\mathcal{S}_{2}$. Si $l_{2} \geq \frac{M}{m}-1$, il est majoré, à une constarte près, par $(-\rho)^{n(1-2 / M)}$.

Cinquième cas.

$$
Y=|\tilde{\mathcal{L}}(\gamma)|^{\frac{2 n i_{1}}{r_{1} i_{2} i_{2}}}|\mathcal{L}(\partial \rho)|^{\frac{2 n}{i_{1} i_{2}+l_{2}}}(-\rho)^{n}\left(\frac{2}{i_{1} i_{2}+i_{2}}\right)
$$

ou 


$$
Y^{\prime}=|\widetilde{\mathcal{L}}(\gamma)|^{\frac{2 n i_{1}}{l_{1} \tau_{2}+l_{2}}}\left|\mathcal{L}\left(\chi_{2}\right)\right|^{\frac{2 n}{l_{1} l_{2}+l_{2}}}(-\rho)^{\frac{2}{\hat{l}_{1} \tilde{l}_{2}+i_{2}}}
$$

pour $\tilde{\mathcal{L}} \in W_{1}$ et $\mathcal{L} \in W_{3}$

Posons $D=l_{1} \tilde{l}_{2}+l_{2}, D^{\prime}=l_{1}\left(\tilde{l}_{2}+1\right)+l_{2}$, et appliquons cette foisci le lemme 3 avec $L=L_{2}$ et avec les paramètres $\alpha=\frac{2 l_{2}}{D}, \beta=\frac{2}{D}$, $A=\frac{2 l_{1}}{D^{\prime}}, B=\frac{2}{D^{\prime}}, A^{\prime}=\frac{2 l_{1}}{D+1}$ et $B^{\prime \prime}=\frac{2}{D+1}$. Il vient

$$
\begin{aligned}
(-\rho)\left|L_{2} Y\right|^{2} \leq\left\{Y+|\widetilde{\mathcal{L}}(\gamma)|^{\frac{2 n l_{1}}{D+1}}\left|L_{2} \mathcal{L}(\partial \rho)\right|^{\frac{2 n}{D+1}}(-\rho)^{n\left(1-\frac{2}{D+1}\right)}+\right. \\
\left.+\left|L_{2} \widetilde{\mathcal{L}}(\gamma)\right|^{\frac{2 n l_{1}}{D^{\prime}}}|\mathcal{L}(\partial \rho)|^{\frac{2 n}{D^{\prime}}}(-\rho)^{n\left(1-\frac{2}{\partial \gamma}\right)}\right\}^{2+\frac{1}{n}}
\end{aligned}
$$

Examinons tout d'abord le deuxième terme de la parenthèse. Si $l_{2}+1<M\left(\frac{m_{l}-l_{2}}{m}\right),\left(L_{2}, \mathcal{L}\right) \in W_{3}$ et ce terme est. une des composantes de $\mathfrak{S}_{2}$. Supposons donc $l_{2}+1 \geq M\left(\frac{m-l_{1}}{m}\right)$ et posons $D^{\prime \prime}=D+1$. Dans ce cas, ce terme est majoré, à unc constante près, par $U=|\widetilde{\mathcal{L}}(\gamma)|^{2 n l_{1} / D^{\prime \prime}}(-\rho)^{n\left(1-2 / D^{\prime \prime}\right)}$. Or, dans $\mathfrak{S}_{2}$ apparait le terme $T=|\widetilde{\mathcal{L}}(\gamma)|^{2 n / i_{2}}(-p)^{n\left(1-2 / i_{2} m\right)}$. Comparons donc $U$ à $T$ :

$$
U=T^{i_{2} t_{1}^{1}}(-\rho)^{\frac{n}{D^{n}}\left(b^{n}-2+\frac{2 t_{1}}{n}-i_{2} l_{1}\right)}
$$

Or, $\mathfrak{S}_{2} \geq(-\rho)^{n(1-2 / M)}$, et, par suite,

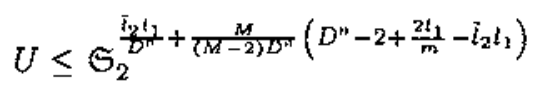

quantité qui est inférieure à $\mathfrak{S}_{2}$ car

$$
\begin{aligned}
(M-2) \tilde{l}_{2} l_{1}+M & \left(D^{\prime \prime}-2+\frac{2 l_{1}}{m}-\tilde{l}_{2} l_{1}\right)= \\
& =M D^{\prime \prime}-2\left(\tilde{l}_{2} l_{1}+M\left(\frac{m-l_{1}}{m}\right)\right) \geq(M-2) D^{\prime \prime}
\end{aligned}
$$

d'après l'hypothèse faite sur $l_{2}$.

Le deuxième terme est donc toujours majoré, à une constante près, par $\mathfrak{S}_{2}$.

Examinons maintenant le troisième tcrme de la parenthèse. Si $\tilde{l}_{2}<\frac{M}{m}-1,\left(L_{2}, \widetilde{\mathcal{L}}\right) \in W_{h}$ et ce terme est une des composantes de $\mathfrak{S}_{2}$. Supposons donc $\tilde{l}_{2} \geq \frac{M}{m}-1$. Il est alors majoré, à une constante près, par $U=|\mathcal{L}(\partial \rho)|^{2 n / D^{\prime}}(-\rho)^{n\left(1-2 / D^{\prime}\right)}$. Nous sommes obligés maintenant de séparer deux cas suivant les valeurs de $l_{2}(\mathcal{L})=l_{2}$. 
Tout d'abord, si $l_{2}>0$, alors $\mathcal{L} \in W_{2}$ et, dans $\mathfrak{S}_{2}$ apparait le terme

$$
T=|\mathcal{L}(\partial \rho)|^{\frac{2 n}{T_{2}}}(-\rho)^{n\left(1-\frac{3}{c}\right)},
$$

avec $c=\frac{l_{2} m}{m-i_{1}}$. Comparons $U$ \ $T$ :

$$
U=T^{\frac{l_{2}}{D^{\prime}}}(-\rho)^{r_{2}\left(1-\frac{2}{D^{\prime}}-\frac{t_{2}}{D^{\prime}}+\frac{2 t_{2}}{c D^{2}}\right)}=\left(T^{l_{2}}(-\rho)^{r_{i}\left(D^{\prime}-l_{2}-\frac{2 t_{1}}{m}\right)}\right)^{\frac{1}{D^{\prime}}}
$$

Or, $\mathfrak{S}_{2} \geq(-p)^{n(1-2 / M)}$, ct par suite

$$
U \leq \mathfrak{S}_{2}^{\frac{D^{\prime} M-2\left(l_{2}+\frac{i_{1} M}{m}\right)}{D^{\prime}\left(M{ }^{2}\right)}}
$$

quantité qui est. inféricure à $S_{2}$ car $\tilde{l}_{2}+1 \geq \frac{M}{m}$ implique $D^{\prime} \geq l_{2}+\frac{M}{m_{m}} l_{\mathrm{j}}$.

Supposons maintenant $l_{2}=0$. Alors $\mathcal{L} \in W_{0}$ ct, dans la somme composant $\boldsymbol{S}_{2}$, apparait le terme

$$
T=|\mathcal{L}(\partial \rho)|^{\frac{2 n}{l_{1}}}(-\rho)^{n\left(1-\frac{2}{t_{1}}+\frac{3}{m}-\frac{2}{m}\right)} .
$$

Comparons $U$ à $T$ :

$$
U=T^{\frac{1}{i_{2}+1}}(-\rho)^{\frac{n}{i_{2}+1}\left(\tilde{l}_{2}-\frac{2}{n}+\frac{2}{M}\right)} .
$$

Comme $S_{2} \geq(-\rho)^{n(1-2 / M)}$ et comme $\tilde{l}_{2} \geq \frac{M}{m}-1$ inplique $\bar{l}_{2}-\frac{2}{m}+\frac{2}{M} \geq 0$, on a

$$
U \leq \mathfrak{S}_{2}^{\frac{M\left(i_{2}+1\right)-2 \frac{M}{m}}{\left(\bar{I}_{2}+1\right)(M-2)}}
$$

quantité qui est inféricure à $\mathfrak{S}_{2}$ car $\frac{M}{m} \leq \tilde{l}_{2}+1$. La démonstration pour $Y^{\prime}$ se fait de façon identique en remplaçant $\mathcal{L}(\partial \rho)$ par $\mathcal{L}\left(\chi_{2}\right)$.

3. Fin de la démonstration de la formule (**).

Nous supposons tout d'abord que $\theta$ est à coefficients $\mathcal{C}^{\infty}$ darns $\bar{\Omega}$.

Comme nous l'avons déjà dit, nous allons appliquer la formule de Stokes sur $\Omega$ cc qui fera apparaître des dérivées des fonctions $\mathcal{G}_{i}$. Le contrôle des dérivées normales obtenu dans la proposition 3 nous amènc à considérer le domaine $\Omega^{\prime}=\Omega \backslash \Omega_{2 \epsilon}$, où $\Omega_{\eta}=\{p<-\eta\}$; ct $0<\epsilon<1$ sera fixé ulériemement.

Pour démontrer la formule (**), il nous faut majorer l'intégrale

$$
\begin{aligned}
I & =\int_{\Omega} \psi \frac{i^{2} \theta \wedge \partial \rho \wedge \bar{\partial} \rho \wedge\left(\omega_{1} \wedge \bar{\omega}_{1}+\omega_{2} \wedge \bar{\omega}_{2}\right)}{\mathfrak{S}_{1}^{1 / n}}+ \\
& +\int_{\Omega} \psi \frac{i^{2} \theta \wedge \partial \rho \wedge \bar{\partial} \rho \wedge \omega_{1} \wedge \bar{\omega}_{1}}{\mathfrak{S}_{2}^{1 / n}}
\end{aligned}
$$


Soit $\varphi \in \mathcal{C}^{\infty}\left(\mathbb{C}^{3}\right), 0 \leq \varphi \leq 1$, telle quc $\varphi \equiv 1$ dans $\Omega \backslash \Omega_{\epsilon}, \operatorname{Supp}(\varphi) \subset$ $\Omega^{\prime}$ et $|\nabla \varphi| \leq C / \epsilon$. Appliquons la formule de Stokes à la forme $\varphi \omega$ où $\omega=(-\rho) \psi \frac{i^{2} \theta \wedge \bar{\partial} \rho \wedge\left(\omega_{1} \wedge \bar{\omega}_{1}+\omega_{2} \wedge \bar{\omega}_{2}\right)}{\mathfrak{S}_{1}^{1 / n}}+(-\rho) \psi \frac{i^{2} \theta \wedge \bar{\partial} \rho \wedge \omega_{1} \wedge \bar{\omega}_{1}}{\mathfrak{S}_{2}^{1 / n}}$.

Puisque $\mathfrak{S}_{i}^{1 / n} \geq(-\rho)^{1-1 / M}, \omega$ est nulle sur $\partial \Omega$ et il vient.

$$
\int_{\Omega} \varphi \partial \omega=-\int_{\Omega} \partial \varphi \wedge \omega
$$

Comme $\mathfrak{S}_{i} \geq \epsilon^{n-2 n / M}$ dans $\Omega_{\epsilon}$, on a $|\omega| \leq \frac{C}{\epsilon^{2}}(-\rho)|\theta|$ dans cet ouvert, et le second membre de (4) est majoré en module par

$$
\frac{C}{\epsilon^{2}} \int_{\Omega}(-\rho)|\theta|
$$

En écrivart $\Omega=\left(\Omega \backslash \Omega_{\epsilon}\right) \cup \Omega_{\epsilon}$ dans les intégrales de $I,(4)$ et $\operatorname{Supp}(\varphi) \subset$ $\Omega^{\prime}$ mortrent que $I$ est inajorée par

$$
I \leq \sum_{k=1}^{4} I_{k}+\frac{C}{\epsilon^{2}} \int_{\Omega}(-\rho)|\theta|
$$

où les $I_{k}$ sont donnés par:

$$
\begin{aligned}
I_{1}= & \int_{\Omega^{\prime}} \frac{(-\rho)}{\mathfrak{S}_{1}^{1 / n}}\left|\partial \psi \wedge \theta \wedge \bar{\partial} \rho \wedge\left(\omega_{1} \wedge \bar{\omega}_{1}+\omega_{2} \wedge \bar{\omega}_{2}\right)\right|+ \\
& +\frac{(-\rho)}{\mathfrak{S}_{2}^{1 / n}}\left|\partial \psi \wedge \theta \wedge \bar{\partial} \rho \wedge \omega_{2} \wedge \bar{\omega}_{2}\right|, \\
I_{2}= & \int_{\Omega^{\prime}} \frac{\psi(-\rho)}{\mathfrak{S}_{1}^{1 / n}}\left|\theta \wedge \partial \bar{\partial} \rho \wedge\left(\omega_{1} \wedge \bar{\omega}_{1}+\omega_{2} \wedge \bar{\omega}_{2}\right)\right|+ \\
& +\frac{\psi(-\rho)}{\mathfrak{S}_{2}^{1 / n}}\left|\theta \wedge \partial \bar{\partial} \rho \wedge \omega_{1} \wedge \bar{\omega}_{1}\right|, \\
I_{3}= & \int_{\Omega^{\prime}} \frac{\psi(-\rho)}{\mathfrak{S}_{1}^{1 / n}}\left|\theta \wedge \bar{\partial} \rho \wedge \partial\left(\omega_{1} \wedge \bar{\omega}_{1}+\omega_{2} \wedge \bar{\omega}_{2}\right)\right|+ \\
& +\frac{\psi(-\rho)}{\mathfrak{S}_{2}^{1 / n}}\left|\theta \wedge \bar{\partial} \rho \wedge \partial\left(\omega_{1} \wedge \bar{\omega}_{1}\right)\right|, \\
I_{1}= & \frac{1}{n} \int_{\Omega^{\prime}} \frac{\psi(-\rho)}{\mathfrak{S}_{1}^{1+1 / n}}\left|\partial \mathfrak{S}_{1} \wedge \theta \wedge \bar{\partial} \rho \wedge\left(\omega_{1} \wedge \bar{\omega}_{1}+\omega_{2} \wedge \bar{\omega}_{2}\right)\right|+ \\
+ & \frac{1}{n} \int_{\Omega^{\prime}} \frac{\psi(-\rho)}{\mathfrak{S}_{2}^{1+1 / n}}\left|\partial \mathfrak{S}_{2} \wedge \theta \wedge \bar{\partial} \rho \wedge \omega_{1} \wedge \bar{\omega}_{1}\right| .
\end{aligned}
$$


Nous allons donc majorer séparément chacune de ces intćgralcs. $\psi$ étant identiqucment nulle à l'extérieur de $B\left(z_{0}, r\right)$, il suffit de considérer comme domaine d'intégration $\Omega^{\prime} \cap B\left(z_{0}, r\right)$ ce qui nous permet d'utiliser les propriétés de $\mathfrak{S}_{1}$ et $\mathfrak{S}_{2}$ établies au paragraphe précédent.

a) Majoration de $I_{1}$ :

Par définition de $\psi, \partial \psi \equiv 0$ sur $B\left(z_{0}, 2 r / 3\right)$, et, d'après la proposition $1, \partial \psi \neq 0$ implique $\mathfrak{S}_{1} \geq 1$ et $\mathfrak{S}_{2} \geq 1 . I_{1}$ est donc majorée, à une constante ne dépendant que de la base $\mathcal{B}$ choisie, de $r$ et de $\psi$ par

$$
I_{1} \leqq \int_{\Omega}(-\rho)|\theta|
$$

b) Majoration de $I_{2}$ :

Développons $\partial \partial \tilde{\rho}$ sur la base de formes associée à $\left(N, L_{1}, L_{2}\right)$ :

$$
\begin{aligned}
\partial \bar{\partial} \rho=a \partial \rho \wedge \bar{\partial} \rho+ & \sum_{i=1}^{2} b_{i} \omega_{i} \wedge \bar{\partial} \rho+\sum_{i=1}^{2} c_{i} \partial \rho \wedge \bar{\omega}_{i}+d \omega_{1} \wedge \bar{\omega}_{2}+e \omega_{2} \wedge \bar{\omega}_{1} \\
& +\partial \rho\left(\left[L_{1}, \bar{L}_{1}\right]\right) \omega_{1} \wedge \bar{\omega}_{1}+\partial \rho\left(\left[L_{2}, \bar{L}_{2}\right]\right) \omega_{2} \wedge \bar{\omega}_{2} .
\end{aligned}
$$

Les termes en $\omega_{i} \wedge \bar{\omega}_{j}, i \neq j$, ne donnant aucune contribution à $I_{2}$, considérons les autres.

$\left.b_{1}\right)$ Contribution du terme $a \partial \rho \wedge \bar{\partial} \rho$ :

$\mathfrak{S}_{i}^{1 / n}$ étant supérieur à $(-\rho)$,

$$
\frac{\left|(-\rho) a \theta \wedge \partial \rho \wedge \bar{\partial} \rho \wedge \omega_{j} \wedge \bar{\omega}_{j}\right|}{\mathfrak{G}_{i}^{1 / n}} \leq C|\theta \wedge \partial \rho \wedge \bar{\partial} \rho|,
$$

où $C$ est une constante ne dépendant que de $B$ et $r$. La contribution de ces termes à $I_{2}$ est donc majorée par $C \int(-\rho)|\theta|$ grâce à la condition de Malliavin (2).

$b_{2}$ ) Contribution des termes $b_{i} \omega_{i} \wedge \bar{\partial} \rho$ :

Les termes $b_{1} \omega_{1} \wedge \bar{\partial} \rho$ et $b_{2} \omega_{2} \wedge \bar{\partial} \rho$ interviennent dans $\theta \wedge \partial \vec{\partial} \rho \wedge\left(\omega_{1} \wedge\right.$ $\left.\bar{\omega}_{1}+\omega_{2} \wedge \bar{\omega}_{2}\right)$ et $b_{2} \omega_{2} \wedge \bar{\partial} \rho$ intervient dans $\theta \wedge \partial \bar{\partial} \rho \wedge \omega_{1} \wedge \bar{\omega}_{1}$. Il nous faut donc majorer

$$
\int_{\Omega^{\prime}} \frac{\psi(-\rho)}{\mathfrak{G}_{1}^{1 / n}}\left(\left|b_{1} \theta \wedge \omega_{2} \wedge \bar{\omega}_{2} \wedge \omega_{1} \wedge \bar{\partial} \rho\right|+\left|b_{2} \theta \wedge \omega_{1} \wedge \bar{\omega}_{1} \wedge \omega_{2} \wedge \bar{\partial} \rho\right|\right)
$$


et,

$$
\int_{\Omega^{\prime}} \frac{\psi(-\rho)}{\mathcal{S}_{2}^{1 / n}}\left|b_{2} \theta \wedge \omega_{1} \wedge \bar{\omega}_{1} \wedge \omega_{2} \wedge \bar{\partial} \rho\right|
$$

Or, puisque $\theta$ est un courant positif, $i \theta \wedge \omega_{j} \wedge \tilde{\omega}_{j}$ est aussi positif, et l'inégalité de Cauchy-Schwarz donne, pour toute constante $K_{1}>0$

$$
\begin{gathered}
\frac{\left|\theta \wedge \omega_{2} \wedge \bar{\omega}_{2} \wedge \omega_{1} \wedge \bar{\partial} \rho\right|}{\mathfrak{S}_{i}^{1 / n}} \leq \\
\leq \frac{1}{K_{1}} \frac{\left|\theta \wedge \omega_{2} \wedge \bar{\omega}_{2} \wedge \partial \rho \wedge \bar{\partial} \rho\right|}{\mathfrak{S}_{i}^{2 / n}}+K_{1}\left|\theta \wedge \omega_{2} \wedge \bar{\omega}_{2} \wedge \omega_{1} \wedge \bar{\omega}_{1}\right|
\end{gathered}
$$

et

$$
\begin{gathered}
\frac{\left|\theta \wedge \omega_{1} \wedge \bar{\omega}_{1} \wedge \omega_{2} \wedge \bar{\partial} \rho\right|}{\mathfrak{S}_{i}^{1 / n}} \leq \\
\leq \frac{1}{K_{1}} \frac{\left|\theta \wedge \omega_{1} \wedge \bar{\omega}_{1} \wedge \partial \rho \wedge \bar{\partial} \rho\right|}{\mathfrak{S}_{i}^{2 / n}}+K_{1}\left|\theta \wedge \omega_{1} \wedge \bar{\omega}_{1} \wedge \omega_{2} \wedge \bar{\omega}_{2}\right| .
\end{gathered}
$$

En utilisant le fait que $\mathfrak{S}_{i}^{2 / n} \geq(-\rho) \mathfrak{S}_{i}^{1 / \pi}$, nous voyons que la contribution de ces termes à $I_{2}$ est inféricure à

$$
\begin{aligned}
C\left\{\frac{1}{K_{1}}\right. & \left\{\int_{\Omega^{\prime}} \psi \frac{\left|\theta \wedge \partial \rho \wedge \bar{\partial} \rho \wedge\left(\omega_{1} \wedge \bar{\omega}_{1}+\omega_{2} \wedge \bar{\omega}_{2}\right)\right|}{\mathfrak{S}_{1}^{1 / \kappa}}+\right. \\
& \left.\left.+\int_{\Omega^{\prime}} \psi \frac{\left|\theta \wedge \partial \rho \wedge \bar{\partial} \rho \wedge \omega_{1} \wedge \bar{\omega}_{1}\right|}{\mathfrak{S}_{2}^{1 / n}}\right\}+K_{1} \int_{\Omega^{\prime}} \psi(-\rho)|\theta|\right\}
\end{aligned}
$$

où la constante $C$ ne dépend que des $b_{i}$ et de $r$, c'est-à-dire du choix de la base $B$ et de $r$.

b3) Contribution des termes $c_{i} \partial \rho \wedge \bar{\omega}_{i}$ :

Ces termes se traitent exactement de la même manière que les précédents.

$\left.b_{1}\right)$ Contribution des termes $\partial \rho\left(\left[L_{1}, \bar{L}_{1}\right]\right) \omega_{1} \wedge \bar{\omega}_{1}$ ct $\partial \rho\left(\left[L_{2}, \bar{L}_{2}\right]\right) \omega_{2} \wedge \bar{\omega}_{2}$ :

D'après la proposition 1, $\mathfrak{G}_{1}^{1 / r} \geq\left|\partial \rho\left(\left[L_{1}, \bar{L}_{1}\right]\right)\right|+\left|\partial \rho\left(\left[L_{2}, \bar{L}_{2}\right]\right)\right|$, et $\mathfrak{S}_{2}^{1 / n} \geq\left|\partial \rho\left(\left[L_{2}, \bar{L}_{2}\right]\right)\right|$. La contribution de ces termes est donc majorćc $\operatorname{par} C \int_{\Omega}(-\rho)|\theta|$. 
Majoration finale de $I_{2}$ :

En regroupant tous ces termes, nous obtenons

$$
I_{2} \lesssim \frac{1}{K_{1}} I+\left(K_{1}+1\right) \int_{\Omega}(-\rho)|\theta|
$$

c) Majoration de $I_{3}$ :

Comme dans le deuxième cas, développons $\partial\left(\omega_{i} \wedge \bar{\omega}_{i}\right)$ sur la base de formes associée à $\left(N, L_{1}, L_{2}\right)$ :

$$
\begin{gathered}
\partial\left(\omega_{i} \wedge \bar{\omega}_{i}\right)=\partial \omega_{i} \wedge \bar{\omega}_{i}-\omega_{i} \wedge \partial \bar{\omega}_{i}=\sum_{j} a_{j} \partial \rho \wedge \omega_{j} \wedge \bar{\omega}_{i}+\sum_{j, k} b_{j, k} \omega_{j} \wedge \omega_{k} \wedge \bar{\omega}_{i}+ \\
+\sum_{j} c_{j} \omega_{i} \wedge \partial \rho \wedge \bar{\omega}_{j}+\sum_{j} d_{j} \omega_{i} \wedge \bar{\partial} \rho \wedge \omega_{j}+\varepsilon \omega_{i} \wedge \partial \rho \wedge \bar{\partial} \rho+\sum_{j} \alpha \alpha_{i j} \omega_{i} \wedge \omega_{j} \wedge \bar{\omega}_{j} .
\end{gathered}
$$

Les termes où apparaît $\bar{\partial} \rho$ donnent unc contribution nulle.

$\left.c_{1}\right)$ Contribution des termes $a_{j} \partial \rho \wedge \omega_{j} \wedge \bar{\omega}_{i}$ et $c_{j} \omega_{i} \wedge \partial \rho \wedge \bar{\omega}_{j}$ :

$\mathfrak{S}_{i}^{1 / \pi}$ étant supérieur à $(-\rho)$,

$$
\frac{\left|(-\rho) a_{j} \theta \wedge \bar{\partial} \rho \wedge \partial \rho \wedge \omega_{j} \wedge \bar{\omega}_{i}\right|}{\mathfrak{S}_{i}^{1 / n}} \leq C|\theta \wedge \partial \rho \wedge \bar{\partial} \rho|
$$

et, comme dans lc cas $b_{1}$ ), en utilisant la condition de Malliavin (2), la contribution de ces termes à l'intégrale $I_{3}$ est majoréc par $C \int_{\Omega}(-\rho)|\theta|$.

La contribution des termes du second type se majore de la même manière.

$c_{2}$ ) Contribution des termes $b_{j, k} \omega_{j} \wedge \omega_{k} \wedge \bar{\omega}_{i}$ :

Ces termes interviennent pour les indices $i=1$ et $i=2$ dans

$$
\frac{\theta \wedge \overline{\bar{\partial}} \rho \wedge \partial\left(\omega_{1} \wedge \bar{\omega}_{1}+\omega_{2} \wedge \bar{\omega}_{2}\right)}{\mathfrak{S}_{1}^{1 / n}}
$$

et, seulement pour l'indice $i=1$ dans

$$
\frac{\left|\theta \wedge \bar{\partial} \rho \wedge \partial\left(\omega_{1} \wedge \bar{\omega}_{1}\right)\right|}{\mathfrak{S}_{2}^{1 / n}} .
$$


Il faut donc dans le premier cas considérer $\omega_{1} \wedge \bar{\omega}_{1} \wedge \omega_{2}$ et $\omega_{2} \wedge \bar{\omega}_{2} \wedge \omega_{1}$ et, daris le second, seulement $\omega_{1} \wedge \bar{\omega}_{1} \wedge \omega_{2}$. Nous devons donc majorer

$$
\begin{array}{r}
\frac{\left|b_{12} \theta \wedge \bar{\partial} \rho \wedge \omega_{1} \wedge \bar{\omega}_{1} \wedge \omega_{2}\right|}{\mathfrak{S}_{1}^{1 / n}}, \frac{\left|b_{21} \theta \wedge \bar{\partial} \rho \wedge \omega_{2} \wedge \bar{\omega}_{2} \wedge \omega_{2}\right|}{\mathfrak{S}_{1}^{1 / n}} \\
\text { et } \frac{\left|b_{12} \theta \wedge \bar{\partial} \rho \wedge \omega_{1} \wedge \bar{\omega}_{1} \wedge \omega_{2}\right|}{\mathfrak{S}_{2}^{1 / n}}
\end{array}
$$

ce qui donne le même résultat que dans le cas $b_{2}$ ) ci-dessus.

c3) Contribution des termes $\alpha_{i j} \omega_{i} \wedge \omega_{j} \wedge \bar{\omega}_{j}$ :

Ces termes apparaissent pour les indices $i=1$ et $i=2$ dans la forme relative ia $S_{1}$ et conduisent à majorer los mône termes (aux coefficients $\alpha_{i j}$ près) que dans les cas $c_{2}$ ) et $b_{2}$ ) ci-dessus.

Par contre, dans la forme relative à $\mathfrak{S}_{2}$ intervient (pour $i=1$ ) la forme $\alpha_{12} \omega_{1} \wedge \omega_{2} \wedge \bar{\omega}_{2}$. La majoration de ce terne nécessite un traitement différent: contrairement aux autres cas, l'application de l'inégalité de Cauchy-Schwarz fait apparaître $\theta \wedge \partial \rho \wedge \bar{\partial} \rho \wedge \omega_{2} \wedge \bar{\omega}_{2}$ avec le poids $\mathfrak{S}_{2}^{-2 / n}$ au heu de $\mathfrak{S}_{1}^{-2 / n}$ et il faut faire intervenir une relation liant $\mathfrak{S}_{1}, \mathfrak{S}_{2}$ et $\alpha_{12}$ (Jusqu'à présent, la seule propriété des coefficients utilisée ćtait qu'ils sont uniformement bornés dans $B\left(z_{0}, r\right)$ par une constante ne dépendant que de la base $B$ et de: $\tau$ ). Nous allons done utiliser la valeur précise de $\alpha_{12}$ :

\section{Lemme 4.}

Le coefficient $-\alpha_{12}$ est égal au conjugué du coefficient $\gamma$ de $L_{1}$ dons la décomposition de $\left[L_{2}, \bar{L}_{2}\right]$ sur la base $\left(N, \bar{N}, L_{1}, \bar{L}_{1}, L_{2}, \bar{L}_{2}\right)$

Démonstration: En effet, $-\alpha_{12} \omega_{1} \wedge \omega_{2} \wedge \bar{\omega}_{2}$ provient du déveioppement de $\omega_{1} \wedge \partial \bar{\omega}_{1}$, et donc $-\alpha_{12}=d \bar{\omega}_{1}\left(L_{2}, \bar{L}_{2}\right)$. Par suite

$$
-\alpha_{12}=L_{2}\left(\bar{\omega}_{1}\left(\bar{L}_{2}\right)\right)-\bar{L}_{2}\left(\bar{\omega}_{1}\left(L_{2}\right)\right)-\bar{\omega}_{1}\left(\left[L_{2}, \bar{L}_{2}\right]\right)=-\bar{\omega}_{1}\left(\left[L_{2}, \bar{L}_{2}\right]\right),
$$

ce qui montre que $\left[L_{2}, \bar{L}_{2}\right]=\alpha_{12} \bar{L}_{1}+b L_{1}$ modulo $\left(L_{2}, \bar{L}_{2}, N, \bar{N}\right)$, et comme le conjugué de $\left[L_{2}, \bar{L}_{2}\right]$ est égal à son opposé, le lemme en résulte.

Nous sommes donc amenés à majorer

$$
\frac{\left|\bar{\gamma} \theta \wedge \bar{\partial} \rho \wedge \omega_{1} \wedge \omega_{2} \wedge \bar{\omega}_{2}\right|}{\mathbb{S}_{2}^{1 / n}} .
$$


$\theta$ étant positif, utilisons l'inégalité de Cauchy-Schwar\% comne on $b_{2}$ ): pour toute constante $K>0$,

$$
\begin{gathered}
\frac{\left|\bar{\gamma} \theta \wedge \bar{\partial} \rho \wedge \omega_{1} \wedge \omega_{2} \wedge \bar{\omega}_{2}\right|}{\mathfrak{S}_{2}^{1 / n}} \leq \\
\leq \frac{1}{K} \frac{|\gamma|^{2}\left|\theta \wedge \partial \rho \wedge \bar{\partial} \rho \wedge \omega_{2} \wedge \bar{\omega}_{2}\right|}{\mathfrak{S}_{2}^{2 / n}}+K\left|\theta \wedge \omega_{1} \wedge \bar{\omega}_{1} \wedge \omega_{2} \wedge \bar{\omega}_{2}\right| .
\end{gathered}
$$

Or, nous avons vu a la proposition 2 que

$$
(-\rho) \frac{|\gamma|^{2}}{\mathfrak{S}_{2}^{2 / n}} \leq \frac{1}{\mathfrak{S}_{1}^{1 / n}}
$$

il vient donc:

$$
\begin{gathered}
\int_{\Omega^{\prime}} \psi(-\rho) \frac{\left|\bar{\gamma} \theta \wedge \bar{\partial} \rho \wedge \omega_{1} \wedge \omega_{2} \wedge \bar{\omega}_{2}\right|}{\mathfrak{S}_{2}^{1 / n}} \leq \\
\leq \frac{1}{K} \int_{\Omega^{\prime}} \psi \frac{\left|\theta \wedge \partial \rho \wedge \bar{\partial} \rho \wedge \omega_{2} \wedge \bar{\omega}_{2}\right|}{\mathfrak{S}_{1}^{1 / n}}+K \int_{\Omega^{\prime}}(-\rho)|\theta| .
\end{gathered}
$$

Majoration finale de $I_{3}$ :

En regroupant les différentes majorations nous obtcrnors:

$$
I_{3} \lesssim \frac{1}{K_{2}} I+\left(K_{2}+1\right) \int_{\Omega}(-\rho)|\theta| .
$$

d) Majoration de $I_{1}$ :

On a

$$
I_{4} \leq \frac{1}{n} \sum_{j=1}^{5} I_{A}^{j},
$$

oì

$$
\begin{aligned}
& I_{a}^{1}=\int_{\Omega^{\prime}} \psi\left|\theta \wedge \partial \rho \wedge \bar{\partial} \rho \wedge\left(\omega_{1} \wedge \bar{\omega}_{1}+\omega_{2} \wedge \bar{\omega}_{2}\right)\right| \frac{(-\rho)\left|N\left(\mathfrak{S}_{1}\right)\right|}{\mathfrak{S}_{1}^{1+1 / n}} \\
& I_{4}^{2}=\int_{\Omega^{\prime}} \psi\left|\theta \wedge \partial \rho \wedge \bar{\partial} \rho \wedge \omega_{1} \wedge \bar{\omega}_{1}\right| \frac{(-\rho)\left|N\left(\mathfrak{S}_{2}\right)\right|}{\mathfrak{S}_{2}^{1+1 / n}} \\
& I_{4}^{3}=\int_{\Omega^{\prime}} \psi\left|\theta \wedge \bar{\partial} \rho \wedge \omega_{1} \wedge \omega_{2} \wedge \bar{\omega}_{2}\right| \frac{(-\rho)\left|L_{1}\left(\mathfrak{S}_{1}\right)\right|}{\mathfrak{S}_{1}^{1+1 / n}} \\
& I_{4}^{4}=\int_{\Omega^{\prime}} \psi\left|\theta \wedge \bar{\partial} \rho \wedge \omega_{1} \wedge \bar{\omega}_{1} \wedge \omega_{2}\right| \frac{(-\rho)\left|L_{2}\left(\mathfrak{S}_{1}\right)\right|}{\mathfrak{S}_{1}^{1+1 / n}}
\end{aligned}
$$

et 


$$
I_{A}^{5}=\int_{\Omega^{\prime}} \psi\left|\theta \wedge \bar{\partial} \rho \wedge \omega_{1} \wedge \bar{\omega}_{1} \wedge \omega_{2}\right| \frac{(-\rho)\left|L_{2}\left(\mathfrak{S}_{2}\right)\right|}{\mathfrak{S}_{2}^{1+1 / n}}
$$

Majorons séparément ces intégrales.

$\left.d_{1}\right)$ D'après la proposition 3 ,

$$
\begin{aligned}
(-\rho)\left|N\left(\mathfrak{S}_{1}\right)\right| & \leq n\left(c_{0}+C(-\rho)^{2 / m}\right) \mathfrak{S}_{1} \\
\text { et }(-\rho)\left|N\left(\mathfrak{S}_{2}\right)\right| & \leq n\left(c_{0}+C(-\rho)^{2 / M}\right) \mathfrak{S}_{2} .
\end{aligned}
$$

Less intcigrales $I_{A}^{1}$ et $I_{A}^{2}$ sont donc majorées par $n\left(c_{0}+C \epsilon^{2 / M}\right) I$.

$\mathrm{d}_{2}$ ) Utilisons à nouveau l'inégalité de Cauchy-Schwarz pour le courant, positif $i \theta \wedge \omega_{j} \wedge \bar{w}_{j}$ :

$$
\begin{aligned}
\left|\theta \wedge \bar{\partial} \rho \wedge \omega_{i} \wedge \omega_{j} \wedge \bar{\omega}_{j}\right| \frac{\left|L_{i}\left(\mathfrak{S}_{k}\right)\right|}{\mathfrak{S}_{k}^{1+1 / n} \leq \frac{1}{K} \mid \theta} & \wedge \partial \rho \wedge \bar{\partial} \rho \wedge \omega_{j} \wedge \bar{\omega}_{j} \mid \frac{\left|L_{i}\left(\mathfrak{S}_{k}\right)\right|^{2}}{\mathfrak{S}_{k}^{2+2 / n}} \\
& +K\left|\theta \wedge \omega_{j} \wedge \bar{\omega}_{j} \wedge \omega_{i} \wedge \bar{\omega}_{i}\right| .
\end{aligned}
$$

La proposition 4 disant, que

$$
(-\rho) \frac{\left|L_{2}\left(\mathfrak{S}_{2}\right)\right|^{2}}{\mathfrak{S}_{2}^{2+2 / n}} \leqslant \frac{1}{\mathfrak{S}_{2}^{1 / n}}, \text { et, }(-\rho) \frac{\left|L_{i}\left(\mathfrak{S}_{1}\right)\right|^{2}}{\mathfrak{S}_{1}^{2+2 / n}} \leqslant \frac{1}{\mathfrak{S}_{1}^{1 / n}}, i=1,2,
$$

chacune des trois dernières intégrales $I_{1}^{k}$ est majorée (a une constante près ne dépendant que de $\mathcal{B}, r$ et $M$ ) par

$$
K \int(-\rho)|\theta|+\frac{1}{K} I
$$

Majoration finale de $I_{4}$ :

En regroupant les différentes majoration des $I_{4}^{k}$ il viert:

$$
I_{1} \leq\left(c_{0}+C \varepsilon^{2 / M}+\frac{C}{K_{3}}\right) I+C\left(K_{3}+1\right) \int_{\Omega}(-\rho)|\theta| .
$$

Fin de la démonstration de la formule (**):

En regroupant les inégalités $(5),(6),(7),(8)$ et $(9)$, il vient:

$$
I \leq\left(c_{0}+\sum_{i=1}^{3} \frac{C}{K_{i}}+C \epsilon^{2 / M}\right) I+\left(\frac{C}{\epsilon^{2}}+C \sum_{i=1}^{3}\left(K_{i}+1\right)\right) \int_{\Omega}(-\rho)|\theta|
$$


et, pour conclure il suffit de choisir les $K_{2}$ assey grands et $\epsilon$ assc petit. de sorte que $\left(c_{0}+\sum_{i=1}^{3} \frac{C}{K_{i}}+C \epsilon^{2 / M}\right)$ soit strictement plus petit que 1 .

Pour terminer complètenient la démonstration de l'négalité $(* *)$, il ne nous reste plus qu'à régulariser le courant positif fermé $\theta$. Or ceci sc fait simplement par convolution avec une suite régularisante, puis on applique le résuitat que nous venons de dénontrer dans $\Omega_{\eta}, \eta>0$, an courant régularisć, et, en faisant tendre $\eta$ vers zóro, on conclut car la constante intervenant dans $(* *)$ pour les courants réguliers ne dépend clairement pas de $\eta$.

\section{Lien avec le multitype de D. Catlin}

Dans ce paragraphe, nous supposerons que $\Omega$ est pseudoconvexe.

Dans [3], D. Catlin introduit le multitype d'un point du bord d'un domaine psendo-convexe borné régulier de $\mathbb{C}^{n}$. En particulier, il montre que si $z_{0} \in \partial \Omega$ il existe une base $\mathcal{B}_{0}=\left(L_{1}^{0}, L_{2}^{0}\right)$ du plan tangent complexe au voisinage de $z_{0}$ tel que le multitype de $z_{0}$ est donné par la non annulation de dérivées de la forme $\mathcal{L}_{B_{0}}(\partial \rho)\left(z_{0}\right)$. Dans ce paragraphe, nous allons donner une estimation des fonctions poids $S\left(L_{1}^{9}, \mathcal{B}_{0}, M\right)(z)$ et. $S\left(L_{2}^{0}, B_{0}, M\right)(z)$ lorsque le point $z$ tend vers $z_{0}$ ch restant sur la normale à $\partial \Omega$ en $z_{0}$.

Nous notons $\left(1, \lambda_{2}, \lambda_{3}\right)$ le multitype de $z_{0}$, et supposons $\lambda_{3}<+\infty$.

Rappelons que, si $\left(Z_{1}, Z_{2}, Z_{3}\right)$ est un système de coordonnées au voisinage de $z_{0}$, oì $Z_{1}$ est porté par lá normale en $z_{0}$, D. Catlin définit dans [3], pour $\Lambda=\left(\mu_{1}, \mu_{2}, \mu_{3}\right)$ et $t>0$, l'ensemble $\mathcal{M}(t, \Lambda)$ des germes de fonctions $\mathcal{C}^{\infty}$ au voisinage de $z_{0}$ telles que $\sum_{i=1}^{3} \frac{\alpha_{i}+\beta_{i}}{\mu_{i}}<t$ entraine $D^{\alpha} \bar{D}^{\beta} f=0$.

En utilisant le fait que si $f \in \mathcal{M}(t, \Lambda)$,

$$
\frac{\partial f}{\partial z_{k}} \in \mathcal{M}\left(t-\frac{1}{\mu_{k}}, \Lambda\right) \text { et } \frac{\partial f}{\partial \bar{z}_{k}} \in \mathcal{M}\left(t-\frac{1}{\mu_{k}}, \Lambda\right)
$$

D. Catlin montre ([3, corollaire 3.4 p. 541]) qu'il cxiste un système de coordonnées pour lequel, pour toute liste $\mathcal{L} \in W(B), f \in \mathcal{M}(t, \Lambda)$ entrainc $\mathcal{L}(f) \in \mathcal{M}\left(t-\frac{l_{1}}{t_{2}}-\frac{l_{2}}{t_{2}}, \Lambda\right)$ et pour lequel (corollaire 3.5 p. 541) $\mathcal{L}(\partial \rho) \in \mathcal{M}\left(1-\frac{l_{1}}{\lambda_{2}}-\frac{l_{2}}{\lambda_{3}},\left(1, \lambda_{2}, \lambda_{3}\right)\right)$ (remarquons que nous ne pouvons déduire ce résultat dircctement de l'égalité entre le mulitype et lo multitype commutateur car dans co dernier les listes sont ordonnées). 
Nous en déduisons que pour toute liste $\mathcal{L}$ de $W, \mathcal{L}(\partial \rho)\left(z_{0}\right)=0$, et, dans un voisinage de $z_{0},|\mathcal{L}(\partial \rho)(z)| \lesssim\left|z-z_{0}\right|$. Si $z$ se déplace sur la normale en $z_{0}$, on a done $|\mathcal{L}(\partial \rho)(z)| \leq(-\rho)$. Alors

$$
\begin{aligned}
& S\left(\mathcal{B}_{0}, \lambda_{2}\right) \text { est ćquivalent à }(-\rho)^{1-\frac{2}{\lambda_{2}}}, \\
& S\left(L_{2}^{0}, \mathcal{B}_{0}, \lambda_{2}, \lambda_{3}\right) \text { est équivalent à }(-\rho)^{1-\frac{2}{\lambda_{3}}} \text {, si } \lambda_{3} \leq 2 \lambda_{2} .
\end{aligned}
$$

Nous allons montrer que ce dernier résultat est encore vrai si $\lambda_{3}>2 \lambda_{2}$. Dans ce cas, l'ensemble des listes $W_{4}$ est non vide et il faut alors estimer $\widetilde{\mathcal{L}}(\gamma)$. Pour simplifier les notations, nous posons, pour la suite, $L_{1}^{0}=L_{1}$, et $L_{2}^{0}=L_{2}$.

Si $\gamma \in \mathcal{M}\left(\frac{1}{\lambda_{2}}-\frac{2}{\lambda_{3}}, \Lambda\right)$, d'après le corollaire 3.4 de [3] et la définition de $\tilde{L}(\gamma)$,

$$
\tilde{\mathcal{L}}(\gamma) \in \mathcal{M}\left(\frac{1}{\lambda_{2}}-\frac{\tilde{l}_{2}}{\lambda_{3}}, \Lambda\right)
$$

Or, pour $\tilde{\mathcal{L}}$ dans $W_{1}\left(L_{2}, \mathcal{B}_{0}, \lambda_{2}, \lambda_{3}\right), i_{2}<\frac{\lambda_{3}}{\lambda_{2}}$ et $\frac{1}{\lambda_{2}}-\frac{i_{2}}{\lambda_{3}}>0$. Il s'ensuit donc que $\tilde{\mathcal{L}}(\gamma)\left(z_{0}\right)=0$ et $S\left(L_{2}, \mathcal{B}, \lambda_{3}, \lambda_{3}\right)$ est bien équivalent $\dot{a}(-p)^{1-2 / \lambda_{3}}$.

Il reste donc à démontrer, que $\gamma \in \mathcal{M}\left(\frac{1}{\lambda_{2}}-\frac{2}{\lambda_{3}}, \Lambda\right)$.

Supposons d'abord $\lambda_{2}>2$. Considérons le boundory system (défini dans $\left[3\right.$, p. 536]) $\mathcal{B}_{2}=\left\{\rho, \rho_{2}, L_{1}\right\}$. En utilisant le fait que $L_{2} \rho_{2} \equiv 0$ (par définition de $L_{2}$ ), nous obtenons

$$
\partial \rho_{2}\left(\left[L_{2}, \bar{L}_{2}\right]\right)=\gamma L_{1} \rho_{2}+\alpha \partial \rho\left(\left[L_{2}, \bar{L}_{2}\right]\right),
$$

où $\alpha$ est. une fonction $\mathcal{C}^{\infty} . L_{1} \rho_{2}$ étant par définition non nul cn $z_{0}$, est inversible dars un voisinage de $z_{0}$ et

$$
\gamma=f \partial \rho_{2}\left(\left[L_{2}, \bar{L}_{2}\right]\right)+g \partial \rho\left(\left[L_{2}, \bar{L}_{2}\right]\right),
$$

oì $f$ ct. $g$ sorit des fonctions $\mathcal{C}^{\infty}$.

Vórifions maintertarit que $\partial \rho_{2}\left(\left[L_{2}, \bar{L}_{2}\right]\right) \in \mathcal{M}\left(\frac{1}{\lambda_{2}}-\frac{2}{\lambda_{2}}, \Lambda\right)$ : cormme le multitype est égnl au commutateur multitype, $\rho \in \mathcal{M}(1, \Lambda)$, et nous pouvons appliquer la proposition 3.6 de $[3$, p.542]. Il s'ensuit que $\rho_{2} \in \mathcal{M}\left(\frac{1}{\lambda_{2}}, \Lambda\right)$ (dans les notations de D. Catlin, $\rho_{2}=r_{2}$ ). Si nous appliquons de roouveau le corollaire 3.5 de $[3]$ à la liste $\left(L_{2}, \bar{L}_{2}\right)$, on obtient le résultat.

Il nous reste à remarquer que $\partial \rho\left(\left[L_{2}, \bar{L}_{2}\right]\right) \in \mathcal{M}\left(\frac{1}{\lambda_{2}}-\frac{2}{\lambda_{3}}, \Lambda\right)$. Or, d'après le corollaire $3.5 \mathrm{de}[3], \partial \rho\left(\left[L_{2}, \bar{L}_{2}\right]\right) \in \mathcal{M}\left(1-\frac{2}{\lambda_{3}}, \Lambda\right)$, ce qui achève la démonstration du cés $\lambda_{2}>2$. 
Considérons maintenant lc cas $\lambda_{2}=2$. Nous utiliserons la propriété suivante $\left(\left[3\right.\right.$, kmme 3.1 p. 538]) : si $f \in \mathcal{M}\left(t_{1}, \Lambda\right)$ et $g \in \mathcal{M}\left(t_{2}, \Lambda\right)$ alors $f g \in \mathcal{M}\left(t_{1}+t_{2}, \Lambda\right)$.

Ecrivons

$$
\left[L_{2}, \bar{L}_{2}\right]=\sum c_{i} \frac{\partial}{\partial z_{i}}+d_{i} \frac{\partial}{\partial \bar{z}_{3}},
$$

( $z_{i}$ ) étant la basc de coordonnćes dans laquelle les corollaires 3.4 et 3.5 de [3] sont vérifiés. La démonstration du corollaire 3.5 de [3, p. 542] montre que $c_{i}$ et $d_{i}$ sont dans $\mathcal{M}\left(\frac{1}{\lambda_{i}}-\frac{2}{\lambda_{3}}, \Lambda\right)$.

Considérons maintenant le crochet $L=\left[\left[L_{2}, \bar{L}_{2}\right], \bar{L}_{1}\right]$. On a dono

$$
L=-\sum \bar{L}_{1} c_{i} \frac{\partial}{\partial z_{i}}+\bar{L}_{1} d_{i} \frac{\partial}{\partial \bar{z}_{i}}+\sum c_{i}\left[\frac{\partial}{\partial z_{i}}, \bar{L}_{1}\right]+\sum d_{i}\left[\frac{\partial}{\partial \bar{z}_{i}}, \bar{L}_{1}\right]
$$

En remarquant que les fonctions $z_{k}$ et $\bar{z}_{k}$ sont dans $\mathcal{M}\left(\frac{1}{\lambda_{k}}, \Lambda\right)$, et en utilisant le corollaire 3.5 de $[3]$, on obtient que, si on écrit

$$
\bar{L}_{1}=\sum \beta_{k} \frac{\partial}{\partial \bar{z}_{k}}
$$

les fonctions $\beta_{k}$ sont dans $\mathcal{M}\left(\frac{1}{\lambda_{k}}-\frac{1}{\lambda_{2}}, \Lambda\right)$.

Estimons alors le coefficient de $\frac{\partial}{\partial z_{i}}$ dans $L$ c'est-ì-dirc $-\bar{L}_{1} c_{2}$. $c_{i} \in \mathcal{M}\left(\frac{1}{\lambda_{i}}-\frac{2}{\lambda_{3}}, \Lambda\right)$ et, d'après le corollaire 3.4 de $[3], \bar{L}_{1} c_{i} \in \mathcal{M}\left(\frac{1}{\lambda_{i}}-\right.$ $\left.\frac{2}{\lambda_{3}}-\frac{1}{2}, \Lambda\right)$.

Puisque $\frac{\partial p}{\partial z_{i}} \in \mathcal{M}\left(1-\frac{1}{\lambda_{i}}, \Lambda\right)$, on a donc $\partial \rho(L) \in \mathcal{M}\left(\frac{1}{2}-\frac{2}{\lambda_{3}}, \Lambda\right)$.

D'autre part,

$$
\left[L_{2}, \bar{L}_{2}\right]=\gamma L_{1}-\gamma L_{1}+\partial \rho\left(\left[L_{2}, \bar{L}_{2}\right]\right) \jmath m N+a L_{2}+b \bar{L}_{2},
$$

d'où

$$
\begin{aligned}
& \left\{\left[L_{2}, \bar{L}_{2}\right] \bar{L}_{1}\right]=\gamma\left[L_{1}, \bar{L}_{1}\right]-\left(\bar{L}_{1} \gamma\right) L_{1}+\left(\bar{L}_{1} \gamma\right) \bar{L}_{1}-\bar{L}_{1}\left(\partial \rho\left(\left[L_{2}, \bar{L}_{2}\right]\right)\right) J_{m N} \\
+ & \partial \rho\left(\left[L_{2}, \bar{L}_{2}\right]\right)\left[I_{m N}, \bar{L}_{1}\right]+\left(\bar{L}_{1} a\right) L_{2}+a\left[L_{2}, \bar{L}_{1}\right]+\left(\bar{L}_{1} b\right) \bar{L}_{2}+b\left[\bar{L}_{2}, \bar{L}_{1}\right]
\end{aligned}
$$

d'où,

$$
\partial \rho(L)=\gamma \partial \rho\left(\left[L_{1}, \bar{L}_{1}\right]\right)+\bar{L}_{1}\left(\partial \rho\left(\left[L_{2}, \bar{L}_{2}\right]\right)\right)+e \partial \rho\left(\left[L_{2}, \bar{L}_{2}\right]\right)+a \partial p\left(\left[L_{2}, \bar{L}_{1}\right]\right),
$$

où $e$ est une fonction $\mathcal{C}^{\infty}$. 
D'après les corollaires 3.4 et 3.5 de $[3]$,

$$
\begin{array}{r}
\partial \rho\left(\left[L_{2}, \bar{L}_{2}\right]\right) \in \mathcal{M}\left(1-\frac{2}{\lambda_{3}}, \Lambda\right), \partial \rho\left(\left[L_{2}, \bar{L}_{1}\right]\right) \in \mathcal{M}\left(1-\frac{1}{2}-\frac{2}{\lambda_{3}}, \Lambda\right), \\
\bar{L}_{1}\left(\partial \rho\left(\left[L_{2}, \bar{L}_{2}\right]\right)\right) \in \mathcal{M}\left(1-\frac{1}{2}-\frac{2}{\lambda_{3}}, \Lambda\right),
\end{array}
$$

et comme $\partial \rho(L)$ est aussi dans $\mathcal{M}\left(\frac{1}{2}-\frac{2}{\lambda_{j}}, \Lambda\right)$, du fait que $\partial \rho\left(\left[L_{1}, \bar{L}_{1}\right]\right)\left(z_{0}\right)$ $\neq 0$, il résulte que $\gamma$ est aussi dans $\mathcal{M}\left(\frac{1}{2}-\frac{2}{\lambda_{3}}, \Lambda\right)$, ce qui achève la démonstration.

Estimation de $S\left(L_{2}^{0}, \mathcal{B}_{0}, M\right)$.

$S\left(\mathcal{B}_{0}, M\right)$ et $S\left(L_{1}^{0}, \mathcal{B}_{0}, m, M\right)$ sont supérieurs à $(-\rho)^{1-2 / M}$, donc, si $M$ cst inférieur à $\lambda_{2}, S\left(L_{1}^{0}, \mathcal{B}_{0}, M\right)$ est supéricur à $(-\rho)^{1-2 / \lambda_{2}}$.

Si $M$ est strictement supérieur à $\lambda_{2}$, toutes les listes $\mathcal{L}$ de longueur $\lambda_{2}$ telłes que $l_{2}(\mathcal{L})=0$ appartiennent à $W_{2}\left(L_{1}^{0}, \mathcal{B}_{0}\right)$ et, par définition de $\lambda_{2}$ il en existe une telle que $\mathcal{L}(\partial \rho)\left(z_{0}\right) \neq 0$. D'oì $S\left(L_{1}^{0}, \mathcal{B}_{0}, m, M\right) \gtrsim$ $(-\rho)^{1-2 / \lambda_{2}}$. La même liste appartient à $W_{1}\left(B_{0}, M\right)$ et on a $S\left(B_{0}, M\right) \gtrsim$ $(-\rho)^{1-2 / \lambda_{2}}$

En rćsumé: si $M$ est supérieur à $\lambda_{2}$ (c'est-à-dire le type de Kohn du point $z_{0}$, lorsque $z$ tend vers $z_{0}$ en restant sur la normale en $z_{0}$,

$$
S\left(L_{1}^{0}, \mathcal{B}_{0}, M\right) \simeq(-\rho)^{1-2 / \lambda_{2}} .
$$

Estimation de $S\left(L_{2}^{0}, B_{0}, M\right)$.

Nous avons vu que $S\left(L_{2}^{0}, B_{0}, \lambda_{2}, \lambda_{3}\right)$ est équivalent à $(-\rho)^{1-2 / \lambda_{3}}$ et que $S\left(L_{2}^{0}, \mathcal{B}_{0}, m, M\right)$ est supérieur à $(-\rho)^{1-2 / M}$. Airsi:

Pour $M \geq \lambda_{3}$, lorsque $z$ tend vers $z_{0}$ en restant sur la normale en $z_{0}$, $S\left(L_{2}^{0}, \mathcal{B}_{0}, M\right)$ vérifie la double inćgalité:

$$
(-\rho)^{1-\frac{3}{M}} \leq S\left(L_{2}^{0}, B_{0}, M\right) \leq(-\rho)^{1-\frac{2}{\lambda_{3}}} .
$$

\section{Références}

1. A. Bonami and Ph. Charpentian, Solutions de l'ćquation $\bar{\partial}$ et zéros de la classe de Nevanlinna dans certains domaines faiblement pscudoconvexes, Ann. Inst. Fourier XXXII, Fasc. 4 (1982), 53-89.

2. A. Bonami and Ph. Charpentier, "Estimations des $(1,1)$-courants positifs fermés dans les domaines de $\mathbb{C}^{2}$, Lecture notes in Math. 1094, 1984, pp. 44-52. 
3. D. Catrin, Boundary invariants of pseudoconvex domains, Annals of Mathematics 120 (1984), 529-586.

4. D.C. Cilang, A. Nagel and E. STein, Estimates for the $\bar{\partial}$-Neumann problem in pseudoconvex domains of finite type, Proc. Natl. Acad. Sci. U.S.A. 85, no. 23 (1988), 8871-8874.

5. A. NAGEL, E. STEIN AND S. WAinger, Boundary bchavior of functions holomorphic in domains of finite type, Proc. Natl. Acad. Sci. U.S.A. 78, no. 11,6596-6599.

6. A. NAGEL, E. STEIN aND S. WaINGER, Balls and metrics defined by vectors fields I: basic properties, Acta Mathematica 155 (1985), 103-147.

\author{
Université de Bordeaux I \\ U.I.IR de Math. Info. \\ 351, cours de la Libération \\ 3340 ' lalence \\ FRANCE
}

Rebut el 20 de Gener de 1992 